\title{
BMJ Open Predictors of hospitalisations for heart failure and mortality in patients with pulmonary hypertension associated with left heart disease: a systematic review
}

\author{
Anastase Dzudie, ${ }^{1,2}$ Andre Pascal Kengne, ${ }^{2,3}$ Friedrich Thienemann, ${ }^{2,4,5}$ \\ Karen Sliwa ${ }^{2,3,6}$
}

To cite: Dzudie A, Kengne AP, Thienemann F, et al. Predictors of hospitalisations for heart failure and mortality in patients with pulmonary hypertension associated with left heart disease: a systematic review. BMJ Open 2014:4:e004843. doi:10.1136/bmjopen-2014004843

- Prepublication history and additional material is available. To view please visit the journal (http://dx.doi.org/ 10.1136/bmjopen-2014004843).

Received 12 January 2014 Revised 4 June 2014 Accepted 17 June 2014

CrossMark

For numbered affiliations see end of article.

Correspondence to Dr Anastase Dzudie; aitdzudie@yahoo.com

\section{ABSTRACT}

Objectives: Left heart disease (LHD) is the main cause of pulmonary hypertension $(\mathrm{PH})$, but little is known regarding the predictors of adverse outcome of $\mathrm{PH}$ associated with LHD (PH-LHD). We conducted a systematic review to investigate the predictors of hospitalisations for heart failure and mortality in patients with PH-LHD.

Design: Systematic review.

Data sources: PubMed MEDLINE and SCOPUS from inception to August 2013 were searched, and citations identified via the ISI Web of Science.

Study selection: Studies that reported on hospitalisation and/or mortality in patients with $\mathrm{PH}$ LHD were included if the age of participants was greater than 18 years and $\mathrm{PH}$ was diagnosed using Doppler echocardiography and/or right heart catheterisation. Two reviewers independently selected studies, assessed their quality and extracted relevant data.

Results: In all, 45 studies (38 from Europe and USA) were included among which $71.1 \%$ were of high quality. 39 studies were published between 2003 and 2013. The number of participants across studies ranged from 46 to 2385; the proportion of men from $21 \%$ to $91 \%$; mean/median age from 63 to 82 years; and prevalence of $\mathrm{PH}$ from $7 \%$ to $83.3 \%$. PH was consistently associated with increased mortality risk in all forms of LHD, except for aortic valve disease where findings were inconsistent. Six of the nine studies with data available on hospitalisations reported a significant adverse effect of $\mathrm{PH}$ on hospitalisation risk. Other predictors of adverse outcome were very broad and heterogeneous including right ventricular dysfunction, functional class, left ventricular function and presence of kidney disease.

Conclusions: $\mathrm{PH}$ is almost invariably associated with increased mortality risk in patients with LHD. However, effects on hospitalisation risk are yet to be fully characterised; while available evidence on the adverse effects of PH have been derived essentially from Caucasians.

\section{Strengths and limitations of this study}

- Our search strategy was likely limited by its focus on a full-report article published in English and French, and traceable via PubMed MEDLINE and/or SCOPUS.

- Important heterogeneity in the included studies precluded the pooling of data to perform a meta-analysis.

- This is the first systematic review on determinants of hospitalisations and mortality in patients with pulmonary hypertension associated with left heart disease, which presents the available up-to-date and high-quality evidence on the subject matter.

\section{INTRODUCTION}

Pulmonary hypertension (PH) describes a group of disorders resulting from an increase in pulmonary vascular resistance, pulmonary blood flow, pulmonary venous pressure or a combination of these features. ${ }^{1}$ Based on shared pathological and haemodynamic characteristics, and therapeutic approaches, five clinical groups of $\mathrm{PH}$ have been distinguished $^{2}$ with $\mathrm{PH}$ associated with left heart disease (PH-LHD) or PH group 2 credited to be the most frequent form of $\mathrm{PH}$ in contemporary clinical settings. ${ }^{3}$ Indeed, $\mathrm{PH}$ is common in patients with LHD, where it often reflects the background LHD, but has also been reported to be a maker of disease severity and unfavourable prognosis. Patients with PH-LHD have more severe symptoms, worse tolerance to effort, experience higher hospitalisation rates and are more likely to receive an indication of the need for cardiac transplant $^{3}$ with major implications for the quality of life of patients and healthcare costs. Several studies have reported PH-LHD 
to be associated with increased mortality, both in patients with systolic dysfunction and those with preserved left ventricular ejection fraction (LVEF). ${ }^{3-6}$ Furthermore, the presence of preoperative $\mathrm{PH}$ has been associated with poor outcomes in patients with valve disease undergoing valve replacement. ${ }^{7}$ However, there are still several gaps in the existing evidence, including the prevalence of PH-LHD and measurement of the true impact of $\mathrm{PH}$ on symptoms and outcome of various LHDs. Equally, little is known regarding the effect of the severity of $\mathrm{PH}$ on hospitalisations, rehospitalisations and death, and their co-factors in patients with LHD. Considering the number of recent advances in the management of $\mathrm{PH}$, it is likely that a better understanding of the impact of PH-LHD on major outcomes might assist the clinical management of patients with $\mathrm{PH}$.

We performed a systematic review of the existing literature to determine the predictors of hospitalisation and mortality in patients with $\mathrm{PH}$ secondary to LHDs including systolic dysfunction, diastolic dysfunction and/or valve disease. Additionally, we aimed to assess whether the severity of $\mathrm{PH}$ affects the risk of the two outcomes.

\section{METHODS}

We searched MEDLINE via PubMed and SCOPUS from inception to August 2013 for all published studies on PH-LHD, using a combination of key words described in the online supplementary box 1 . All searches were restricted to studies in humans published in 'English' or 'French' languages. In addition, we manually searched the reference lists of eligible studies and relevant reviews, and traced studies that had cited them through the ISI Web of Science for any relevant published and unpublished data. Two independent reviewers (AD and APK) performed the study selection, data extraction and quality assessment; and disagreements were resolved by consensus or consulting a third reviewer (KS).

Studies that reported on hospitalisation and/or mortality in patients with PH-LHD were included if the following criteria were met: (1) age of participants greater than 18 years; (2) Right ventricular systolic pressure (RVSP) measured by transthoracic Doppler echocardiography (DE) and calculated from the maximum tricuspid regurgitation jet velocity using the modified Bernoulli equation $\left(4 v^{2}\right)$ and adding right atrial pressure (RAP). RAP could be a fixed value from 5 to $10 \mathrm{~mm} \mathrm{Hg}$, could have been estimated clinically using the jugular venous pressure (JVP), or estimated by measuring the inferior vena cava size and change with spontaneous respiration using echocardiography; and/or (3) mean pulmonary artery pressure (mPAP) measured by right heart catheterisation (RHC) or by DE. We excluded narrative reviews and case series. Studies on persistent $\mathrm{PH}$ following heart transplantation were not included because of the complexity of the classification of $\mathrm{PH}$ in this population.
The following variables were extracted from each study: publication year, country of origin of the study, study design, study population's demographics, the mean/median follow-up duration, the outcome predicted, the proportion of measurable RVSP, the mean/ median baseline RVSP or mPAP, the prevalence of PH, the readmission rate, the mortality rate with odds ratio (OR) or hazard ratio (HR) for $\mathrm{PH}$ where reported and the predictors of outcome including the tricuspid annular plan systolic excursion (TAPSE). One study ${ }^{8}$ reported the effect of $\mathrm{PH}$ in relation with survival. Effects on mortality were obtained by taking the inverse of the HR for survival.

\section{Quality assessment}

The methodological quality of the selected studies was assessed using the Quality In Prognosis Studies (QUIPS) tool, designed for systematic reviews of prognostic studies through an international expert consensus (table 1). ${ }^{52}$ The QUIPS contains six domains assessing the following: (1) bias due to patient selection; (2) attrition; (3) measurement of prognostic factors; (4) outcome measurement; (5) confounding on statistical analysis and reporting results; and (6) confounding on presentation. In prognosis studies designed to predict a specific outcome based on a combination of several possible prognostic factors, confounding is not an issue. Therefore, the items on confounding were considered irrelevant for our quality assessment. The remaining 17 items of the five categories each were scored to assess the quality of the included studies. For each study, the five domains were scored separately as high $(+)$, moderate $( \pm$ ) or low (-) quality (ie, presenting a low, moderate or high risk of bias, respectively). To strengthen the discriminative capacity of the QUIPS, we used the scoring algorithm developed by de Jonge et $a l l^{53}$ as explained, described in detail in the online supplementary table.

\section{Data synthesis}

Hospitalisations or rehospitalisations for heart failure and mortality identified by multivariable analysis in individual studies are presented (table 2), including their estimated effect size (eg, OR or HR) and 95\% CI. Quantitative analysis of results was not done due to important heterogeneity in study design, study population, $\mathrm{PH}$ definition and measurement, outcome definitions in the studies and confounding or other types of prognostic factors. We have therefore presented a narrative summary of the available evidence (table 2).

\section{RESULTS}

\section{Studies selection}

Figure 1 presents a flow diagram for the study selection process. Of the 7550 citations identified through searches, 6255 titles were examined and 6083 were excluded on the basis of the title scanning. The remaining 172 abstracts were examined and 55 articles were 


\begin{tabular}{|c|c|c|c|c|c|c|c|c|c|c|c|}
\hline $\mathbf{N}$ & Study & Country/ethnicity & Design & $\begin{array}{l}\text { Statistical } \\
\text { methods }\end{array}$ & $\begin{array}{l}\text { Study } \\
\text { participation }\end{array}$ & $\begin{array}{l}\text { Study } \\
\text { attrition }\end{array}$ & $\begin{array}{l}\text { Measurement of } \\
\text { prognostic } \\
\text { factors }\end{array}$ & $\begin{array}{l}\text { Assessment of } \\
\text { outcomes }\end{array}$ & $\begin{array}{l}\text { Statistical } \\
\text { analysis and } \\
\text { presentation }\end{array}$ & $\begin{array}{l}\text { Quality } \\
\text { score } \\
\text { (points) }\end{array}$ & $\begin{array}{l}\text { Quality: } \\
+=\text { high } \\
\text { 土=moderate } \\
\text {-=low }\end{array}$ \\
\hline 1 & Merlos et $a l^{9}$ & Spain & $\begin{array}{l}\text { Prospective hospital } \\
\text { based cohort }\end{array}$ & $\mathrm{KM}$, Cox regression & 13.5 & 15 & 10 & 15 & 15 & 68.5 & + \\
\hline 2 & Agarwal et al ${ }^{10}$ & $\begin{array}{l}\text { USA - ethnicity data in } \\
98 \text { patients ( } 63 \% \text { whites) }\end{array}$ & $\begin{array}{l}\text { Retrospective hospital } \\
\text { based cohort }\end{array}$ & $\mathrm{KM}$, Cox regression & 13.5 & 7.5 & 12.5 & 15 & 15 & 63.5 & + \\
\hline 3 & Agarwal $^{11}$ & USA-96\% blacks & $\begin{array}{l}\text { Prospective hospital } \\
\text { based cohort }\end{array}$ & $\mathrm{KM}$, Cox regression & 12 & 10 & 10 & 15 & 15 & 62 & + \\
\hline 4 & Aronson et al ${ }^{12}$ & USA & $\begin{array}{l}\text { Prospective hospital } \\
\text { based cohort }\end{array}$ & Cox regression & 15 & 15 & 15 & 15 & 12.5 & 72.5 & + \\
\hline 5 & Bursi et $a l^{13}$ & $\begin{array}{l}\text { USA } \\
\text { Caucasians and blacks }\end{array}$ & $\begin{array}{l}\text { Prospective population } \\
\text { based cohort study }\end{array}$ & $\begin{array}{l}\mathrm{KM} \text {, Logistic } \\
\text { regression }\end{array}$ & 15 & 12.5 & 12.5 & 12.5 & 15 & 65 & + \\
\hline 6 & Strange et al ${ }^{14}$ & Armadale-Australia & $\begin{array}{l}\text { Retrospective population } \\
\text { based cohort }\end{array}$ & $\begin{array}{l}\mathrm{KM} \text {, Logistic and } \\
\text { Cox regression }\end{array}$ & 15 & 7.5 & 10 & 12.5 & 12.5 & 58.5 & \pm \\
\hline 7 & Mutlak et $a l^{15}$ & USA & $\begin{array}{l}\text { Prospective hospital } \\
\text { based cohort }\end{array}$ & $\begin{array}{l}\mathrm{KM} \text {, Logistic and } \\
\text { Cox regression, } \\
\mathrm{KM}\end{array}$ & 13.5 & 15 & 10 & 15 & 15 & 69 & + \\
\hline 8 & Tatebe et al ${ }^{16}$ & Japan & $\begin{array}{l}\text { Prospective hospital } \\
\text { based cohort }\end{array}$ & $\begin{array}{l}\mathrm{KM} \text {, Logistic and } \\
\text { Cox regression }\end{array}$ & 15 & 10 & 15 & 15 & 15 & 72.5 & + \\
\hline 9 & Adhyapak et $a l^{8}$ & India & $\begin{array}{l}\text { Prospective hospital } \\
\text { based cohort }\end{array}$ & Cox regression & 13.5 & 10 & 10 & 12.5 & 5 & 53.5 & \pm \\
\hline 10 & Stern et al ${ }^{17}$ & USA & $\begin{array}{l}\text { Retrospective hospital } \\
\text { based cohort }\end{array}$ & $\mathrm{KM}$, Cox regression & 13.5 & 15 & 12.5 & 12.5 & 12.5 & 66 & + \\
\hline 11 & Lee et al ${ }^{18}$ & Korea & $\begin{array}{l}\text { Prospective hospital } \\
\text { based cohort }\end{array}$ & $\mathrm{KM}$, Cox regression & 15 & 15 & 15 & 12.5 & 15 & 72.5 & + \\
\hline 12 & Møller et al ${ }^{19}$ & USA & $\begin{array}{l}\text { Prospective hospital } \\
\text { based cohort }\end{array}$ & $\begin{array}{l}\text { KM, Logistic } \\
\text { regression }\end{array}$ & 13.5 & 15 & 12.5 & 15 & 15 & 71 & + \\
\hline 13 & Cappola et al ${ }^{20}$ & $\begin{array}{l}\text { USA, } 35 \% \text { blacks and } \\
65 \% \text { whites }\end{array}$ & $\begin{array}{l}\text { Prospective hospital } \\
\text { based cohort }\end{array}$ & $\mathrm{KM}$, Cox regression & 13.5 & 7.5 & 12.5 & 15 & 15 & 62.5 & + \\
\hline 14 & Szwejkowski et al ${ }^{21}$ & UK & $\begin{array}{l}\text { Retrospective hospital } \\
\text { based cohort }\end{array}$ & $\mathrm{KM}$, Cox regression & 13.5 & 10 & 10 & 15 & 15 & 61 & + \\
\hline 15 & Abramson et al ${ }^{22}$ & USA & $\begin{array}{l}\text { Prospective hospital } \\
\text { based cohort }\end{array}$ & $\mathrm{KM}$, Cox regression & 12 & 15 & 10 & 15 & 12.5 & 64.5 & + \\
\hline 16 & Kjaergaard et al ${ }^{23}$ & Denmark & $\begin{array}{l}\text { Prospective hospital } \\
\text { based cohort }\end{array}$ & $\mathrm{KM}$, Cox regression & 13.5 & 15 & 12.5 & 15 & 15 & 71 & + \\
\hline 17 & Shalaby et $a l^{24}$ & USA, 95\% Caucasians & $\begin{array}{l}\text { Retrospective hospital } \\
\text { based cohort }\end{array}$ & $\mathrm{KM}$, Cox regression & 13.5 & 12.5 & 15 & 15 & 15 & 71 & + \\
\hline 18 & Damy et $a^{25}$ & UK & $\begin{array}{l}\text { Prospective hospital } \\
\text { based cohort }\end{array}$ & $\begin{array}{l}\mathrm{KM} \text {, Logistic and } \\
\text { Cox regression }\end{array}$ & 15 & 10 & 15 & 15 & 15 & 70 & + \\
\hline 19 & Ristow et $a l^{26}$ & USA & $\begin{array}{l}\text { Prospective hospital } \\
\text { based cohort }\end{array}$ & Logistic regression & 13.5 & 12.5 & 10 & 15 & 5 & 48.5 & \pm \\
\hline 20 & Grigioni et al ${ }^{27}$ & Italy & Retrospective cohort & $\begin{array}{l}\text { KM, Logistic } \\
\text { regression }\end{array}$ & 13.5 & 12.5 & 12.5 & 15 & 15 & 68.5 & \pm \\
\hline 21 & Levine et $a l^{28}$ & $\begin{array}{l}\text { USA, mainly Caucasians } \\
(78.3 \%)\end{array}$ & Retrospective cohort & $\begin{array}{l}\text { No Logistic } \\
\text { regression, no KM } \\
\text { analysis }\end{array}$ & 12 & 10 & 10 & 7.5 & 2.5 & 42 & - \\
\hline 22 & Lam et $a l^{29}$ & USA & $\begin{array}{l}\text { Prospective observational } \\
\text { community based cohort }\end{array}$ & $\begin{array}{l}\text { KM, Logistic } \\
\text { regression }\end{array}$ & 12 & 15 & 10 & 15 & 12.5 & 68 & + \\
\hline 23 & Khush et al ${ }^{30}$ & $\begin{array}{l}\text { Multicentric USA and } \\
\text { Canada }\end{array}$ & $\begin{array}{l}\text { Prospective cohort in the } \\
\text { ESCAPE trial }\end{array}$ & $\mathrm{KM}$ & 15 & 10 & 15 & 15 & 12.5 & 68.5 & + \\
\hline
\end{tabular}




\begin{tabular}{|c|c|c|c|c|c|c|c|c|c|c|c|}
\hline $\mathbf{N}$ & Study & Country/ethnicity & Design & $\begin{array}{l}\text { Statistical } \\
\text { methods }\end{array}$ & $\begin{array}{l}\text { Study } \\
\text { participation }\end{array}$ & $\begin{array}{l}\text { Study } \\
\text { attrition }\end{array}$ & $\begin{array}{l}\text { Measurement of } \\
\text { prognostic } \\
\text { factors }\end{array}$ & $\begin{array}{l}\text { Assessment of } \\
\text { outcomes }\end{array}$ & $\begin{array}{l}\text { Statistical } \\
\text { analysis and } \\
\text { presentation }\end{array}$ & $\begin{array}{l}\text { Quality } \\
\text { score } \\
\text { (points) }\end{array}$ & $\begin{array}{l}\text { Quality: } \\
\text { +=high } \\
\text { 土=moderate } \\
\text {-=low }\end{array}$ \\
\hline 24 & Ghio et $a^{31}$ & Italy & Prospective cohort & $\mathrm{KM}$, Cox regression & 13.5 & 12.5 & 12.5 & 12.5 & 12.5 & 63.5 & + \\
\hline 25 & Wang et $a l^{32}$ & China & Retrospective cohort & KM & 12 & 12.5 & 12.5 & 12.5 & 5 & 54.5 & \pm \\
\hline 26 & Ghio et $a^{33}$ & Italy & Prospective cohort & $\begin{array}{l}\text { KM, Cox and } \\
\text { Logistic regression }\end{array}$ & 13.5 & 10 & 10 & 15 & 15 & 63.5 & + \\
\hline 27 & Naidoo et $a^{34}$ & South Africa, Blacks & Retrospective cohort & $\begin{array}{l}\text { No Logistic } \\
\text { regression, no } \\
\text { Kaplan Meier } \\
\text { analysis }\end{array}$ & 12 & 7.5 & 10 & 5 & 7.5 & 42 & - \\
\hline 28 & Fawzy et al ${ }^{35}$ & Saudi Arabia & Prospective cohort & $\begin{array}{l}\text { No Logistic } \\
\text { regression, no } \\
\text { Kaplan Meier }\end{array}$ & 12 & 10 & 12.5 & 15 & 7.5 & 57 & \pm \\
\hline 29 & Roseli et $a l^{36}$ & USA & $\begin{array}{l}\text { Retrospective hospital } \\
\text { based cohort }\end{array}$ & $\mathrm{KM}$, Cox regression & 13.5 & 10 & 10 & 15 & 12.5 & 63.5 & \pm \\
\hline 30 & Melby et $a l^{37}$ & USA & $\begin{array}{l}\text { Retrospective hospital } \\
\text { based cohort }\end{array}$ & $\mathrm{KM}$, Cox regression & 13.5 & 12.5 & 10 & 15 & 15 & 66 & + \\
\hline 31 & Le Tourneauet al ${ }^{38}$ & $\begin{array}{l}\text { France, mainly } \\
\text { Caucasians }\end{array}$ & $\begin{array}{l}\text { Prospective hospital } \\
\text { based cohort }\end{array}$ & $\mathrm{KM}$, Cox regression & 13.5 & 10 & 10 & 15 & 15 & 63.5 & + \\
\hline 32 & Parker et $\mathrm{al}^{7}$ & USA & $\begin{array}{l}\text { Retrospective hospital } \\
\text { based cohort }\end{array}$ & $\mathrm{KM}$, Cox regression & 12 & 15 & 12.5 & 15 & 15 & 71 & + \\
\hline 33 & Kainuma et al ${ }^{39}$ & Japan, Asians & $\begin{array}{l}\text { Retrospective hospital } \\
\text { based cohort }\end{array}$ & $\mathrm{KM}$, Cox regression & 10.5 & 10 & 12.5 & 12.5 & 10 & 55.5 & \pm \\
\hline 34 & Barbieri et al ${ }^{40}$ & $\begin{array}{l}\text { Multicentric (Europe and } \\
\text { USA) }\end{array}$ & $\begin{array}{l}\text { Prospective hospital } \\
\text { based cohort }\end{array}$ & $\mathrm{KM}$, Cox regression & 13.5 & 15 & 12.5 & 15 & 15 & 71 & + \\
\hline 35 & Manners et al ${ }^{41}$ & United Kingdom & $\begin{array}{l}\text { Retrospective hospital } \\
\text { based cohort }\end{array}$ & $\begin{array}{l}\text { No regression } \\
\text { analysis, no KM } \\
\text { estimation }\end{array}$ & 10.5 & 7.5 & 5 & 5 & 2.5 & 30.5 & - \\
\hline 36 & Malouf et al ${ }^{42}$ & USA & $\begin{array}{l}\text { Prospective hospital } \\
\text { based cohort }\end{array}$ & $\begin{array}{l}\text { KM, Cox and } \\
\text { Logistic regression }\end{array}$ & 10.5 & 10 & 10 & 15 & 12.5 & 58 & + \\
\hline 37 & Khandhar et al ${ }^{43}$ & USA & $\begin{array}{l}\text { Retrospective hospital } \\
\text { based cohort }\end{array}$ & $\mathrm{KM}$, Cox regression & 13.5 & 10 & 10 & 15 & 12.5 & 61 & \pm \\
\hline 38 & Zuern et al ${ }^{44}$ & Germany & $\begin{array}{l}\text { Prospective hospital } \\
\text { based cohort }\end{array}$ & $\mathrm{KM}$, Cox regression & 15 & 7.5 & 10 & 15 & 15 & 62.5 & + \\
\hline 39 & Ben-Dor et al ${ }^{45}$ & USA & $\begin{array}{l}\text { Prospective hospital } \\
\text { based cohort }\end{array}$ & $\begin{array}{l}\text { KM, Logistic } \\
\text { regression }\end{array}$ & 15 & 10 & 10 & 15 & 15 & 68 & + \\
\hline 40 & Yang et $a l^{46}$ & USA & $\begin{array}{l}\text { Retrospective hospital } \\
\text { based cohort }\end{array}$ & $\begin{array}{l}\mathrm{KM} \text {, Cox and } \\
\text { logistic regression }\end{array}$ & 15 & 7.5 & 15 & 12.5 & 15 & 65 & + \\
\hline 41 & Nozohoor et al ${ }^{47}$ & Sweden & Retrospective cohort & $\begin{array}{l}\text { KM, Cox and } \\
\text { Logistic regression }\end{array}$ & 13.5 & 10 & 10 & 15 & 12.5 & 61 & + \\
\hline 42 & $\begin{array}{l}\text { Ward and } \\
\text { Hancock }\end{array}$ & UK & Retrospective cohort & $\begin{array}{l}\text { No KM, no Logistic } \\
\text { or Cox regression }\end{array}$ & 12 & 5 & 2.5 & 7.5 & 2.5 & 29.5 & - \\
\hline 43 & Ghoreishi et al ${ }^{49}$ & USA & Retrospective cohort & $\begin{array}{l}\text { KM, Cox and } \\
\text { Logistic regression }\end{array}$ & 15 & 10 & 10 & 10 & 15 & 60 & + \\
\hline 44 & Cam et al ${ }^{50}$ & USA & Retrospective cohort & $\begin{array}{l}\text { KM, Cox and } \\
\text { Logistic regression }\end{array}$ & 13.5 & 15 & 10 & 10 & 12.5 & 61 & + \\
\hline 45 & Pai et $a l^{51}$ & USA & Retrospective cohort & $\begin{array}{l}\mathrm{KM}, \text { Cox and } \\
\text { Logistic regression }\end{array}$ & 15 & 10 & 10 & 10 & 15 & 60 & + \\
\hline
\end{tabular}

KM, Kaplan Meier. 


\begin{tabular}{|c|c|c|c|c|c|c|c|c|c|c|c|c|c|c|}
\hline \multirow[b]{2}{*}{$\begin{array}{l}\text { Author, year } \\
\text { published }\end{array}$} & \multirow{2}{*}{$\begin{array}{l}\text { Diagnostic criteria } \\
\text { (RVSP by } \\
\text { echocardiography } \\
\text { or mPAP by } \\
\text { echocardiography } \\
\text { or RHC) }\end{array}$} & \multirow{2}{*}{$\begin{array}{l}\text { Study population (sample } \\
\text { size, heart disease, } \\
\text { NYHA class, type of HF) }\end{array}$} & \multirow[b]{2}{*}{$\begin{array}{l}\text { Mean/ } \\
\text { median } \\
\text { follow-up } \\
\text { (months) }\end{array}$} & \multirow[b]{2}{*}{$\begin{array}{l}\text { Age-years/ } \\
\text { male sex-\% }\end{array}$} & \multirow[b]{2}{*}{$\begin{array}{l}\text { Definition of } \\
\text { outcomes } \\
\text { predicted }\end{array}$} & \multirow{2}{*}{$\begin{array}{l}\text { Proportion } \\
\text { (\%) of } \\
\text { measurable } \\
\text { RVSP }\end{array}$} & \multirow{2}{*}{$\begin{array}{l}\text { Median/mean } \\
(\mathrm{mm} \mathrm{Hg}) \\
\text { baseline } \\
\text { RVSP (echo) } \\
\text { or mPAP } \\
\text { (RHC) }\end{array}$} & \multirow[b]{2}{*}{$\begin{array}{l}\text { Prevalence } \\
\text { of } \mathrm{PH} \text { at } \\
\text { baseline } \\
(\%)\end{array}$} & \multirow{2}{*}{$\begin{array}{l}\text { HF } \\
\text { readmission } \\
\text { rate or } \\
\text { adjusted } \\
\text { ORs/HRs } \\
\text { and } \mathrm{Cl}\end{array}$} & \multicolumn{4}{|c|}{$\begin{array}{l}\text { Mortality (all-cause) rate at } 6,12,24 \text { and } 36 \text { months or } \\
\text { at mean duration of follow-up }\end{array}$} & \multirow{2}{*}{$\begin{array}{l}\text { Adjusted ORs/ } \\
\text { HRs and } \mathrm{Cl} \text { (or } \\
\text { p value) for } \\
\text { all-cause } \\
\text { mortality, } \\
\text { outcome }\end{array}$} \\
\hline & & & & & & & & & & 6 & 12 & 24 & $\begin{array}{l}36 \text { or at } \\
\text { mean/ } \\
\text { median } \\
\text { follow-up }\end{array}$ & \\
\hline \multicolumn{15}{|c|}{ Studies in patients with heart failure and cardiomyopathies } \\
\hline $\begin{array}{l}\text { Merlos et al, } \\
2013^{9}\end{array}$ & RVSP >35 mm Hg & $\begin{array}{l}1210 \text { consecutive patients } \\
\text { with HF, stratified into } \\
\text { normal (RVSP <35), mild } \\
\text { (RVSP } 36-45) \text {, moderate } \\
\text { (RVSP 46-60) and severe } \\
\text { PH (RVSP >60 mm Hg) }\end{array}$ & 12 & $\begin{array}{l}72.6 \\
54.1 \%\end{array}$ & $\begin{array}{l}\text { All-cause mortality } \\
\text { Cardiovascular } \\
\text { deaths }\end{array}$ & 41.5 & 46 & 35.2 & NR & NR & $\begin{array}{l}4.89 / 10 \\
\text { persons-year } \\
\text { in severe } \mathrm{PH}\end{array}$ & NA & NR & $\begin{array}{l}\text { OR for mild PH } \\
1.6(0.7 \text { to } 3.74) \text {, } \\
\text { moderate } \mathrm{PH} \\
1.34(0.54 \text { to } \\
3.16) \text { and severe } \\
\mathrm{PH} 2.57(1.07 \text { to } \\
6.27)\end{array}$ \\
\hline $\begin{array}{l}\text { Agawal et al, } \\
2012^{10}\end{array}$ & $\begin{array}{l}\text { RHC with mPAP } \\
>25 \mathrm{~mm} \mathrm{Hg}\end{array}$ & $\begin{array}{l}339 \text { patients with } \mathrm{PH} \text { and } \\
\text { LHD, } 90 \% \text { with } \mathrm{HFpEF} \text {, } \\
\text { NYHA class NR }\end{array}$ & 54.2 & $63 / 21 \%$ & All-cause mortality & NA & 43 & NA & NR & NR & $2.9 \%$ & $4.4 \%$ & $6.8 \%$ & $\begin{array}{l}\text { UTSW cohort HR } \\
1.4(1.1 \text { to } 1.9) \\
\text { and NU cohort } \\
\text { HR } 1.4 \text { (1.1 to } \\
1.7)\end{array}$ \\
\hline $\begin{array}{l}\text { Agawal, } \\
2012^{11}\end{array}$ & RVSP $>35$ & $\begin{array}{l}288 \text { patients undergoing } \\
\text { haemodialysis stratified into } \\
\text { PH and NPH- based on } \\
\text { RVSP }\end{array}$ & 25.8 & $\begin{array}{l}56.5 \text { vs } 53.1 / \\
65 \text { vs } 63 \%\end{array}$ & All-cause mortality & NA & 44.7 vs 27.2 & 38 & NR & NR & 26.4 vs 24.5 & 48.3 vs 46.3 & 62.9 vs 56.3 & $\begin{array}{l}\text { HR } 2.17 \text { (1.31 to } \\
3.61)\end{array}$ \\
\hline $\begin{array}{l}\text { Aronson } \\
\text { et al, } 2011^{12}\end{array}$ & $\begin{array}{l}\text { RHC with mPAP } \\
\geq 25 \mathrm{~mm} \mathrm{Hg} \text { and } \\
\mathrm{mPCWP} \\
>15 \mathrm{~mm} \mathrm{Hg}\end{array}$ & $\begin{array}{l}242 \text { patients with acute HF, } \\
\text { divided in } 3 \text { groups, NPH, } \\
\text { passive PH and reactive } \\
\text { PH, NYHA class IV }\end{array}$ & 6 & $61 ; 42 \%$ & All-cause mortality & NA & 34 vs 38 vs 44 & 76.0 & NR & $\begin{array}{l}8.6 \text { vs } 21 \\
\text { vs } 48.3\end{array}$ & NR & NR & NR & $\begin{array}{l}\text { HR for passive } \\
\text { PH } 1.7(0.6 \text { to } \\
4.5) \text { and reactive } \\
\text { PH } 4.8 \text { (2.1 to } \\
17.5)\end{array}$ \\
\hline $\begin{array}{l}\text { Bursi et al, } \\
2012^{13}\end{array}$ & RVSP >35 mm Hg & $\begin{array}{l}1049 \text { patients with HF } \\
\text { stratified into tertiles of } \\
\text { RVSP }(<41,41-54 \text { and } \\
>54 \mathrm{~mm} \mathrm{Hg})\end{array}$ & 81 & $76 ; 49.3 \%$ & All-cause mortality & NR & 48 & 79 & NA & NR & $\begin{array}{l}4,10, \text { and } \\
17 \% \text { for } \\
\text { tertiles } 1,2, \\
\text { and } 3, \\
\text { respectively }\end{array}$ & 8 vs 19 vs 28 & 46 & $\begin{array}{l}\text { HR for tertile } 2: \\
1.45(1.13 \text { to } \\
1.85) \text { and tertile } \\
3: 2.07(1.62 \text { to } \\
2.64)\end{array}$ \\
\hline $\begin{array}{l}\text { Strange } \\
\text { et } a l, 2012^{14}\end{array}$ & $\mathrm{RVSP}>40 \mathrm{~mm} \mathrm{Hg}$ & $\begin{array}{l}15633 \text { echo screening, } 636 \\
\text { PH group } 2 \text { stratified into } 3 \\
\text { groups (group } 1 \text { RVSP } \\
<40 \mathrm{~mm} \mathrm{Hg} \text {, group } 2 \\
\text { between } 41 \text { and } 60 \text { and } \\
\text { group } 3>60 \mathrm{~mm} \mathrm{Hg} \text { ) }\end{array}$ & 83 & $79 ; 48 \%$ & All-cause mortality & NR & 52 & NR & NA & NR & NR & NR & $\begin{array}{l}\text { Mean } \\
\text { survival } \\
4.2 \text { years }\end{array}$ & NR \\
\hline $\begin{array}{l}\text { Mutlak et al, } \\
2012^{15}\end{array}$ & RVSP > 35 mm Hg & $\begin{array}{l}1054 \text { patients with acute } \\
\text { myocardial infarction } \\
\text { divided into NPH and PH } \\
\text { groups }\end{array}$ & 12 & $\begin{array}{l}60 \text { vs } 69 \\
77 \text { vs } 64 \%\end{array}$ & $\begin{array}{l}\text { Readmission for HF } \\
\text { All-cause mortality }\end{array}$ & NR & 32 vs 43 & 44.6 & $\begin{array}{l}2.1 \text { vs } 9.2 ; \\
\text { OR } 3.1(1.87 \\
\text { to } 5.14)\end{array}$ & NR & NR & NR & NR & $\begin{array}{l}\text { HR for } \\
\text { readmission } 3.1 \\
\text { (1.87 to } 5.14)\end{array}$ \\
\hline $\begin{array}{l}\text { Tatebe et al, } \\
2012^{16}\end{array}$ & $\begin{array}{l}\text { RHC with mPAP } \\
\geq 25 \mathrm{~mm} \mathrm{Hg} \\
\mathrm{mPCWP} \\
>15 \mathrm{~mm} \mathrm{Hg}\end{array}$ & $\begin{array}{l}676 \text { consecutive patients } \\
\text { with chronic HF, NYHA } \\
\text { class } \geq 2 \text {, stratified into } 3 \\
\text { groups, NPH (mPAP }<25 \text { ), } \\
\text { passive PH (PH with PVR } \\
\geq 2.5 \text { WU) or reactive PH } \\
\text { (PH with PVR }>2.5 \text { WU) }\end{array}$ & 31.2 & $\begin{array}{l}\text { 64vs 64vs 63; } \\
\text { 63vs 48vs } 66 \%\end{array}$ & $\begin{array}{l}\text { All-cause mortality } \\
\text { and readmission for } \\
\text { HF }\end{array}$ & NR & $\begin{array}{l}17 \text { vs } 30 \text { vs } 35 \\
\text { in } \mathrm{NPH}, \\
\text { passive } \mathrm{PH} \\
\text { and reactive } \\
\mathrm{PH} \text {, } \\
\text { respectively }\end{array}$ & 23 & NR & NR & $\begin{array}{l}24.5 \text { vs } 18 \text { vs } \\
18.9 \% \text { in } \\
\mathrm{NPH} \text {, passive } \\
\text { and reactive } \\
\mathrm{PH}, \\
\text { respectively }\end{array}$ & $\begin{array}{l}52.5 \text { vs } 50 \text { vs } \\
60.3 \% \text { in } \mathrm{NPH}, \\
\text { passive and } \\
\text { reactive } \mathrm{PH}, \\
\text { respectively }\end{array}$ & $\begin{array}{l}71.0 \text { vs } 77 \\
\text { vs } 79.3 \text { in } \\
\mathrm{NPH}, \\
\text { passive PH } \\
\text { and reactive } \\
\mathrm{PH}, \\
\text { respectively }\end{array}$ & $\begin{array}{l}\text { HR for reactive } \\
\text { PH group } 1.18 \\
\text { (1.03 to } 1.35)\end{array}$ \\
\hline $\begin{array}{l}\text { Adhyapak, } \\
2010^{8}\end{array}$ & $\begin{array}{l}\text { Echocardiography } \\
\text { with mPAP } \\
>25 \mathrm{~mm} \mathrm{Hg}\end{array}$ & $\begin{array}{l}147 \text { patients with HF } \\
\text { stratified into: group 1, } \\
\text { normal PASP/preserved } \\
\text { RV function; group 2, } \\
\text { normal PASP/RV } \\
\text { dysfunction; group 3, } \\
\text { high PASP/preserved RV } \\
\text { function; and group 4, high } \\
\text { PASP/RV dysfunction }\end{array}$ & 11.2 & $\begin{array}{l}54 \\
91.8 \%\end{array}$ & $\begin{array}{l}\text { Cardiac death } \\
\text { Readmissions }\end{array}$ & NR & $\begin{array}{l}\text { Group } 120 \pm 5 \\
\text { group } 224.8 \\
\pm 0.4 \text { group } 3 \\
56.8 \pm 6 \text { and } \\
\text { group } 458.9 \\
\pm 8.8\end{array}$ & 53.7 & $\begin{array}{l}\text { 19.7, OR and } \\
\text { CINR }\end{array}$ & & $\begin{array}{l}\text { Overall } 5.1 \text { at } \\
11.2 \text { months, } \\
4.5 \text { in group } \\
3 \text { vs } 8.8 \text { in } \\
\text { group } 4\end{array}$ & NA & NA & $\begin{array}{l}\text { HR in PH } 2.27 \\
\text { (1.09 to } 3.57)\end{array}$ \\
\hline
\end{tabular}




\begin{tabular}{|c|c|c|c|c|c|c|c|c|c|c|c|c|c|c|}
\hline \multirow[b]{2}{*}{$\begin{array}{l}\text { Author, year } \\
\text { published }\end{array}$} & \multirow{2}{*}{$\begin{array}{l}\text { Diagnostic criteria } \\
\text { (RVSP by } \\
\text { echocardiography } \\
\text { or mPAP by } \\
\text { echocardiography } \\
\text { or RHC) } \\
\end{array}$} & \multirow{2}{*}{$\begin{array}{l}\text { Study population (sample } \\
\text { size, heart disease, } \\
\text { NYHA class, type of HF) }\end{array}$} & \multirow[b]{2}{*}{$\begin{array}{l}\text { Mean/ } \\
\text { median } \\
\text { follow-up } \\
\text { (months) }\end{array}$} & \multirow[b]{2}{*}{$\begin{array}{l}\text { Age-years/ } \\
\text { male sex-\% }\end{array}$} & \multirow[b]{2}{*}{$\begin{array}{l}\text { Definition of } \\
\text { outcomes } \\
\text { predicted }\end{array}$} & \multirow{2}{*}{$\begin{array}{l}\text { Proportion } \\
\text { (\%) of } \\
\text { measurable } \\
\text { RVSP }\end{array}$} & \multirow{2}{*}{$\begin{array}{l}\text { Median/mean } \\
(\mathrm{mm} \mathrm{Hg}) \\
\text { baseline } \\
\text { RVSP (echo) } \\
\text { or mPAP } \\
\text { (RHC) }\end{array}$} & \multirow[b]{2}{*}{$\begin{array}{l}\text { Prevalence } \\
\text { of PH at } \\
\text { baseline } \\
(\%)\end{array}$} & \multirow{2}{*}{$\begin{array}{l}\text { HF } \\
\text { readmission } \\
\text { rate or } \\
\text { adjusted } \\
\text { ORs/HRs } \\
\text { and } \mathrm{Cl}\end{array}$} & \multicolumn{4}{|c|}{$\begin{array}{l}\text { Mortality (all-cause) rate at 6, } 12,24 \text { and } 36 \text { months or } \\
\text { at mean duration of follow-up }\end{array}$} & \multirow{2}{*}{$\begin{array}{l}\text { Adjusted ORs/ } \\
\text { HRs and Cl (or } \\
\text { p value) for } \\
\text { all-cause } \\
\text { mortality, } \\
\text { outcome }\end{array}$} \\
\hline & & & & & & & & & & 6 & 12 & 24 & $\begin{array}{l}\text { mean/ } \\
\text { median } \\
\text { follow-up }\end{array}$ & \\
\hline $\begin{array}{l}\text { Stern et al, } \\
2007^{17}\end{array}$ & $\begin{array}{l}\text { Echocardiography } \\
\text { but criteria for PH } \\
\text { not reported }\end{array}$ & $\begin{array}{l}68 \text { patients needing } \\
\text { cardiac resynchronisation } \\
\text { stratified into group } 1 \\
\text { (RVSP } \geq 50 \mathrm{~mm} \mathrm{Hg}, \mathrm{n}=27 \text { ) } \\
\text { and group } 2(\mathrm{RVSP} \\
<50 \mathrm{~mm} \mathrm{Hg}, \mathrm{n}=41 \text { ) }\end{array}$ & 7.1 & $\begin{array}{l}70 \\
64.7 \%\end{array}$ & $\begin{array}{l}\text { Composite of } \\
\text { hospitalisation for } \\
\text { HF and all-cause } \\
\text { mortality }\end{array}$ & NR & $\begin{array}{l}\text { Group } 139.7 \\
\pm 6.7 \text { and group } \\
260.2 \pm 9.2\end{array}$ & NR & $\mathrm{NR}$ & NR & $\begin{array}{l}\text { Increased } \\
\text { mortality in } \\
\text { patients with } \\
\text { RVSP } \\
\geq 50 \mathrm{~mm} \mathrm{Hg}\end{array}$ & NR & NR & $\begin{array}{l}\text { HR of } 2.0 \text { ( } 1.2 \text { to } \\
5.5) \text { for RVSP } \\
\geq 50\end{array}$ \\
\hline $\begin{array}{l}\text { Lee et al, } \\
2010^{18}\end{array}$ & RVSP >39 mm Hg & $\begin{array}{l}813 \text { patients with TR } \\
\text { stratified into two groups } \\
\text { based on the RVSP } \\
<39 \mathrm{~mm} \mathrm{Hg} \text { (group 1, } \\
\mathrm{n}=530 \text { ) and RVSP } \\
\geq 39 \mathrm{~mm} \mathrm{Hg} \text { (group 2, } \\
\mathrm{n}=283 \text { ) }\end{array}$ & 58.8 & $\begin{array}{l}64 \\
42.5 \%\end{array}$ & All-cause mortality & NR & $\begin{array}{l}37.1 \text { in patients } \\
\text { who survived } \\
\text { vs } 43.8 \text { in } \\
\text { patients who } \\
\text { died }\end{array}$ & NR & NR & NR & NR & 10.5 vs 21.9 & $\begin{array}{l}\text { 5-year } \\
\text { survival } \\
\text { rates } 61.0 \\
\text { and } 80.6 \% \\
\text { group 2 vs } \\
\text { group 1 } \\
\text { respectively }\end{array}$ & $\begin{array}{l}\text { HR of } 1.024 \\
\text { (1.017 to } 1.032)\end{array}$ \\
\hline $\begin{array}{l}\text { Møller et al, } \\
2005^{19}\end{array}$ & RVSP > 30 mm Hg & $\begin{array}{l}536 \text { patients with acute } \\
\text { myocardial infarction } \\
\text { stratified into group } 1 \\
\text { (RVSP }<30 \mathrm{~mm} \mathrm{Hg} \text { ), group } \\
2 \text { mild to moderate } \mathrm{PH} \\
\text { (RVSP of } 31 \text { to } 55 \mathrm{~mm} \mathrm{Hg} \text { ) } \\
\text { and group } 3 \text { severe } \mathrm{PH} \\
\text { (RVSP }>55 \mathrm{~mm} \mathrm{Hg} \text { ) }\end{array}$ & 40 & $\begin{array}{l}65 / 68 \% \\
74 / 54 \% \\
78 / 44 \% \text { in } \\
\text { groups } 1,2 \\
\text { and } 3, \\
\text { respectively }\end{array}$ & All-cause mortality & 69 & NR & 75 & NR & NR & NR & $\begin{array}{l}5 \% \text { in group } 1 \\
52 \% \text { in } \\
\text { patients with a } \\
\text { RVSP } \\
>65 \mathrm{~mm} \mathrm{Hg}\end{array}$ & NR & $\begin{array}{l}\text { HR } 1.22(1.14 \text { to } \\
1.38) \text { per } \\
10 \mathrm{~mm} \mathrm{Hg} \\
\text { increased }\end{array}$ \\
\hline $\begin{array}{l}\text { Cappola } \\
\text { et al, } 2012^{20}\end{array}$ & $\begin{array}{l}\text { RHC with mPAP } \\
\geq 25 \mathrm{~mm} \mathrm{Hg}\end{array}$ & $\begin{array}{l}1134 \text { patients with } \\
\text { cardiomyopathy stratified } \\
\text { according to PVR: NPH } \\
\text { (<2.5), group } 1 \mathrm{PH}(2.5-3) \text {, } \\
\text { group } 2 \mathrm{PH}(3-3.5) \text {, group } \\
3 \mathrm{PH}(3.5-4) \text { and group } 4 \\
\mathrm{PH}(>4)\end{array}$ & 52.8 & $\begin{array}{l}48 \\
60 \%\end{array}$ & All-cause mortality & NA & 25 & NR & NR & NR & NR & $\mathrm{NR}$ & $\begin{array}{l}33 \% \text { of } \\
\text { patients died } \\
\text { during the } \\
\text { mean FU }\end{array}$ & $\begin{array}{l}\text { HR } 1.86 \text { (1.30 to } \\
2.65) \text { for group } 2, \\
1.78(1.13 \text { to } \\
2.81) \text { for group } 3 \\
\text { and } 2.04 \text { (1.51 to } \\
2.74) \text { for group } 4\end{array}$ \\
\hline $\begin{array}{l}\text { Szwejkowski } \\
\text { et al, } 2011^{21}\end{array}$ & RVSP $>33 \mathrm{~mm} \mathrm{Hg}$ & $\begin{array}{l}1612 \text { patients with HF } \\
\text { stratified into } 5 \text { groups } \\
\text { according to RVSP (<33; } \\
33-38 ; 39-44 ; 45-52 \text { and } \\
>52 \mathrm{~mm} \mathrm{Hg} \text { ) }\end{array}$ & 33.6 & $\begin{array}{l}75.2 \\
57.4 \%\end{array}$ & All-cause mortality & 32 & 46 & 83.3 & NR & NR & NR & NR & $\begin{array}{l}55.1 \% \text { of } \\
\text { patients died } \\
\text { during the } \\
\text { mean FU }\end{array}$ & $\begin{array}{l}\text { HR } 1.06(1.03 \text { to } \\
1.08) \text { for every } \\
5 \mathrm{~mm} \mathrm{Hg} \\
\text { increase in } \\
\text { RVSP }\end{array}$ \\
\hline $\begin{array}{l}\text { Abramson } \\
\text { et al, } 1992^{22}\end{array}$ & $\begin{array}{l}\text { Echocardiography } \\
\text { with TRV }>2.5 \mathrm{~m} / \mathrm{s}\end{array}$ & $\begin{array}{l}108 \text { patients with dilated } \\
\text { cardiomyopathy, stratified } \\
\text { into } 2 \text { groups: group } 1 \\
\text { (TRV }<2.5 \mathrm{~m} / \mathrm{s} \text { ) and group } \\
2(>2.5 \mathrm{~m} / \mathrm{s}), 38.9 \% \text { in } \\
\text { NYHA class III and IV, } \\
77.3 \% \text { of ischaemic HF }\end{array}$ & 28 & $\begin{array}{l}67.5 \\
81 \%\end{array}$ & $\begin{array}{l}\text { All-cause mortality, } \\
\text { mortality due to HF } \\
\text { and } \\
\text { re-hospitalisations } \\
\text { for HF }\end{array}$ & NR & $5.6 \mathrm{~m} / \mathrm{s}$ & 26 & $\begin{array}{l}75 \% \text { during } \\
\text { the study } \\
\text { period } \\
5.76(1.97 \text { to } \\
16.90)\end{array}$ & NR & NR & NR & $\begin{array}{l}17 \% \text { in } \\
28 \text { months } \\
\text { vs } 57 \%\end{array}$ & $\begin{array}{l}\text { OR for increased } \\
\text { TRV } 3.77(1.38 \\
\text { to } 10.24)\end{array}$ \\
\hline $\begin{array}{l}\text { Kjaergaard } \\
\text { et al, } 2007^{23}\end{array}$ & $\begin{array}{l}\text { Echocardiography } \\
\text { but cut-off for PH } \\
\text { not reported }\end{array}$ & $\begin{array}{l}388 \text { consecutive patients } \\
\text { with known or presumed } \\
\text { HF stratified into quartiles } \\
\text { of RVSP }(<31,31-38,39- \\
50,>50)\end{array}$ & 33.6 & $\begin{array}{l}75 \\
60 \%\end{array}$ & All-cause mortality & NR & 38 & $\begin{array}{l}75 \% \text { and } \\
50 \% \text { with } \\
\text { RVSP }>31 \\
\text { and } \\
40 \mathrm{~mm} \mathrm{Hg} \text {, } \\
\text { respectively }\end{array}$ & NR & & $\begin{array}{l}48 \% \text { if COPD } \\
\text { and } 21 \% \text { in } \\
\text { HF without } \\
\text { COPD }\end{array}$ & NR & $\begin{array}{l}57 \% \text { at } \\
33.6 \text { months }\end{array}$ & $\begin{array}{l}\text { HR } 1.09 \text { (1.04 to } \\
\text { 1.14) for every } \\
\text { increase of } \\
\text { RVSP per } \\
5 \mathrm{~mm} \mathrm{Hg}\end{array}$ \\
\hline $\begin{array}{l}\text { Shalaby } \\
\text { et al, } 2008^{24}\end{array}$ & $\mathrm{RVSP} \geq 30 \mathrm{~mm} \mathrm{Hg}$ & $\begin{array}{l}270 \text { patients undergoing } \\
\text { cardiac resynchronisation } \\
\text { stratified into } 3 \text { groups on } \\
\text { the basis of RVSP: group } \\
1,(22-29, n=86) \text {; group } 2 \\
(30-44, n=90) \text { and group } 3 \\
(45-88, n=94) \text {. }\end{array}$ & 19.4 & $\begin{array}{l}66.5 \\
91 \%\end{array}$ & $\begin{array}{l}\text { All-cause mortality, } \\
\text { cardiac } \\
\text { transplantation } \\
\text { (primary end point) } \\
\text { or re-hospitalisation } \\
\text { for HF }\end{array}$ & NR & 40.4 & NR & $\begin{array}{l}40 \% \text { in group } \\
3 \text { vs } 9 \% \text { in } \\
\text { group } 1(6.35 \\
(2.55 \text { to } \\
15.79))\end{array}$ & NR & NR & NR & $\begin{array}{l}12 \% \text { in } \\
\text { group } 1 \% \text { vs } \\
34 \% \text { in } \\
\text { group } 3 \text { at } \\
\text { mean } \\
\text { follow-up }\end{array}$ & $\begin{array}{l}\text { HR } 2.62(1.07 \text { to } \\
6.41)\end{array}$ \\
\hline
\end{tabular}




\begin{tabular}{|c|c|c|c|c|c|c|c|c|c|c|c|c|c|c|}
\hline \multirow[b]{2}{*}{$\begin{array}{l}\text { Author, year } \\
\text { published }\end{array}$} & \multirow{2}{*}{$\begin{array}{l}\text { Diagnostic criteria } \\
\text { (RVSP by } \\
\text { echocardiography } \\
\text { or mPAP by } \\
\text { echocardiography } \\
\text { or RHC) } \\
\end{array}$} & \multirow{2}{*}{$\begin{array}{l}\text { Study population (sample } \\
\text { size, heart disease, } \\
\text { NYHA class, type of HF) }\end{array}$} & \multirow[b]{2}{*}{$\begin{array}{l}\text { Mean/ } \\
\text { median } \\
\text { follow-up } \\
\text { (months) } \\
\end{array}$} & \multirow[b]{2}{*}{$\begin{array}{l}\text { Age-years/ } \\
\text { male sex-\% }\end{array}$} & \multirow[b]{2}{*}{$\begin{array}{l}\text { Definition of } \\
\text { outcomes } \\
\text { predicted }\end{array}$} & \multirow{2}{*}{$\begin{array}{l}\text { Proportion } \\
(\%) \text { of } \\
\text { measurable } \\
\text { RVSP } \\
\end{array}$} & \multirow{2}{*}{$\begin{array}{l}\text { Median/mean } \\
(\mathrm{mm} \mathrm{Hg}) \\
\text { baseline } \\
\text { RVSP (echo) } \\
\text { or mPAP } \\
\text { (RHC) } \\
\end{array}$} & \multirow[b]{2}{*}{$\begin{array}{l}\text { Prevalence } \\
\text { of PH at } \\
\text { baseline } \\
(\%)\end{array}$} & \multirow{2}{*}{$\begin{array}{l}\text { HF } \\
\text { readmission } \\
\text { rate or } \\
\text { adjusted } \\
\text { ORs/HRs } \\
\text { and } \mathrm{Cl} \\
\end{array}$} & \multicolumn{4}{|c|}{$\begin{array}{l}\text { Mortality (all-cause) rate at } 6,12,24 \text { and } 36 \text { months or } \\
\text { at mean duration of follow-up }\end{array}$} & \multirow{2}{*}{$\begin{array}{l}\text { Adjusted ORs/ } \\
\text { HRs and } \mathrm{Cl} \text { (or } \\
\mathrm{p} \text { value) for } \\
\text { all-cause } \\
\text { mortality, } \\
\text { outcome }\end{array}$} \\
\hline & & & & & & & & & & 6 & 12 & 24 & $\begin{array}{l}\text { mean/ } \\
\text { median } \\
\text { follow-up }\end{array}$ & \\
\hline $\begin{array}{l}\text { Damy et al, } \\
2010^{25}\end{array}$ & $\begin{array}{l}\text { Echocardiography } \\
\text { with RVTG } \\
>25 \mathrm{~mm} \mathrm{Hg}\end{array}$ & $\begin{array}{l}1380 \text { patients with } \\
\text { congestive HF, } 1026 \text { with } \\
\text { LVSD (EF <45\%) and } 324 \\
\text { without), further stratified } \\
\text { into quartiles of RVSP }\end{array}$ & 66 & $\begin{array}{l}72 \\
67 \%\end{array}$ & All-cause mortality & $\begin{array}{l}30 \% \text { of all, } \\
26 \% \text { in } \\
\text { patients with } \\
\text { LVSD and } \\
40 \% \text { in those } \\
\text { without }\end{array}$ & 25 & $\begin{array}{l}46 \% \text { of } \\
\mathrm{HFpEF}, 50 \% \\
\text { of } \mathrm{HFrEF} \\
\text { and } 23 \% \text { of } \\
\text { patients } \\
\text { without HF }\end{array}$ & $\begin{array}{l}\text { NA } \\
\text { (outpatient } \\
\text { cohort) }\end{array}$ & NR & NR & NR & $\begin{array}{l}40.3 \% \text { at } \\
\text { median } \\
\text { follow-up of } \\
66 \text { months }\end{array}$ & $\begin{array}{l}\text { HR } 1.72(1.16 \text { to } \\
2.55) \text { for RVSP } \\
>45 \mathrm{~mm} \mathrm{Hg})\end{array}$ \\
\hline $\begin{array}{l}\text { Ristow et al, } \\
2007^{26}\end{array}$ & $\begin{array}{l}\text { Echocardiography } \\
\text { with TR gradient } \\
>30 \mathrm{~mm} \mathrm{Hg}\end{array}$ & $\begin{array}{l}717 \text { patients with coronary } \\
\text { artery disease, } 573 \text { with } \\
\text { measurable TR, stratified } \\
\text { into group } 1 \text { (TR gradient } \\
\leq 30 \mathrm{~mm} \mathrm{Hg}, \mathrm{n}=447) \text { and } \\
\text { group } 2(\mathrm{TR} \text { gradient } \\
>30 \mathrm{~mm} \mathrm{Hg}, \mathrm{n}=126)\end{array}$ & 36 & $\begin{array}{l}65,74 \% \\
\text { (group 1) 69, } \\
75 \% \text { (group 2) }\end{array}$ & $\begin{array}{l}\text { Hospitalisation, CV } \\
\text { death, all-cause } \\
\text { death and the } \\
\text { combined end point } \\
\text { of all }\end{array}$ & 80 & NR & 22 & $\begin{array}{l}6 \% \text { (group I) } \\
\text { vs } 21 \% \\
\text { (group II) OR } \\
\text { per each } \\
10 \mathrm{~mm} \mathrm{Hg} \\
\text { increase of } \\
\text { TR gradient } \\
1.5(1.03 \text { to } \\
2.2 \text { ) }\end{array}$ & NR & NR & NR & $\begin{array}{l}11 \% \text { (group } \\
\text { 1) vs } 17 \% \\
\text { (group 2) }\end{array}$ & $\begin{array}{l}\text { OR for all-cause } \\
\text { deaths } 1.2(0.85 \\
\text { to } 1.6) \text { per } \\
10 \mathrm{~mm} \mathrm{Hg} \\
\text { increase in TR } \\
\text { OR for combined } \\
\text { endpoint } 1.6(1.1 \\
\text { to } 2.4)\end{array}$ \\
\hline $\begin{array}{l}\text { Grigioni et al, } \\
2006^{27}\end{array}$ & $\begin{array}{l}\text { RHC with mPAP } \\
\geq 25 \mathrm{~mm} \mathrm{Hg}\end{array}$ & $\begin{array}{l}196 \text { patients with HF } \\
\text { evaluated for PH and } \\
\text { changes in mPAP }\end{array}$ & 24 & $\begin{array}{l}54 \\
73 \%\end{array}$ & $\begin{array}{l}\text { Cardiovascular } \\
\text { deaths, acute HF } \\
\text { and combined end } \\
\text { point of both }\end{array}$ & NA & 25 & NR & $\begin{array}{l}27 \% \text { acute } \\
\text { HF, } 2.30 \\
\text { (1.42 to } 3.73 \text { ) }\end{array}$ & NR & NR & $\begin{array}{l}20 \% \\
\text { cardiovascular } \\
\text { deaths }\end{array}$ & $\mathrm{NR}$ & $\begin{array}{l}\text { HR for PH } 2.3 \\
(1.42 \text { to } 3.73) ; \\
\text { HR for worsening } \\
>30 \% \text { in mPAP } \\
2.6(1.45 \text { to } 4.67)\end{array}$ \\
\hline $\begin{array}{l}\text { Levine et al, } \\
1996^{28}\end{array}$ & $\begin{array}{l}\text { RHC assessed } \\
\text { change in } \mathrm{PH}, \text { no } \\
\text { definition }\end{array}$ & $\begin{array}{l}60 \text { patients with PH owing } \\
\text { to } \mathrm{HF} \text { awaiting heart } \\
\text { transplantation, stratified } \\
\text { into } 2 \text { groups: group } A \\
\text { (persistent elevated sPAP, } \\
\mathrm{n}=31 \text { ), group } \mathrm{B} \text { (decrease } \\
\text { in } \mathrm{sPAP}, \mathrm{n}=29 \text { ) }\end{array}$ & 10 & $\begin{array}{l}50 \\
85 \%\end{array}$ & $\begin{array}{l}\text { Transplant or } \\
\text { all-cause death }\end{array}$ & NA & $\begin{array}{l}39 \text { vs } 57 \text { in } \\
\text { group } A \text { and } \\
\text { group B, } \\
\text { respectively }\end{array}$ & NA & NR & NR & NR & NR & $\begin{array}{l}90 \% \text { vs } 50 \% \\
\text { of death at } \\
10 \text { months in } \\
\text { group A and } \\
\text { group B, } \\
\text { respectively }\end{array}$ & NR \\
\hline $\begin{array}{l}\text { Lam al, } \\
2010^{29}\end{array}$ & RVSP >35 mm Hg & $\begin{array}{l}244 \text { patients with HFpEF } \\
\text { compared with } 719 \\
\text { subjects with HTN. } 203 \\
\text { patients with HFpEF and } \\
\text { PH later stratified into: } \\
\text { group } 1 \text { (RVSP } \\
<48 \mathrm{~mm} \mathrm{Hg} \text { ) and group } 2 \\
(\mathrm{RVSP}>48 \mathrm{~mm} \mathrm{Hg})\end{array}$ & 33.6 & $\begin{array}{l}74 / 47 \% \text { vs } 79^{*} / \\
41 \% \text { in group } 1 \\
\text { and group } 2, \\
\text { respectively }\end{array}$ & All-cause mortality & $\begin{array}{l}65 \text { vs } 83 \% \text { in } \\
\text { HTN and } \\
\text { HFpEF, } \\
\text { respectively }\end{array}$ & $\begin{array}{l}28 \mathrm{vs} \\
48 \mathrm{~mm} \mathrm{Hg} \text { in } \\
\mathrm{HTN} \text { and } \\
\mathrm{HFpEF} \text {, } \\
\text { respectively }\end{array}$ & $\begin{array}{l}8 \text { vs } 83 \% \text { in } \\
\text { HTN and } \\
\text { HFpEF, } \\
\text { respectively }\end{array}$ & NR & NR & $\begin{array}{l}12.2 \text { vs } 25.7 \\
\text { in group } 1 \\
\text { and group } 2 \text {, } \\
\text { respectively }\end{array}$ & $\begin{array}{l}18.4 \text { vs } 36.2 \text { in } \\
\text { group } 1 \text { and } \\
\text { group 2, } \\
\text { respectively }\end{array}$ & $\begin{array}{l}55.1 \text { vs } 63.8 \\
\text { in group } 1 \\
\text { and group } 2 \text {, } \\
\text { respectively }\end{array}$ & $\begin{array}{l}\text { HR } 1.20 \text { per } \\
\text { each increase of } \\
10 \mathrm{~mm} \mathrm{Hg} \text { in } \\
\operatorname{RVSP}(p<0.001)\end{array}$ \\
\hline $\begin{array}{l}\text { Kush et al, } \\
2009^{30}\end{array}$ & $\begin{array}{l}\text { RHC with mixed PH } \\
\text { (MPH) defined as } \\
\text { mPAP } \geq 25 \mathrm{~mm} \mathrm{Hg} \text {, } \\
\text { PCWP }>15 \mathrm{~mm} \mathrm{Hg} \text {, } \\
\text { and PVR } \geq 3 \mathrm{WU}\end{array}$ & $\begin{array}{l}171 \text { patients with severe } \\
\text { HFrEF (NYHA class IV, } \\
\text { LVEF } \leq 30 \% \text {,systolic BP } \\
\leq 125 \mathrm{~mm} \mathrm{Hg} \text { ) further } \\
\text { stratified into } 2 \text { groups: } \\
\text { MPH group (mPAP } \\
>25 \mathrm{~mm} \mathrm{Hg} \text { and PVR }>3 \\
\text { WU, } n=80 \text { ) and non-MPH } \\
(\mathrm{mPAP}<25 \mathrm{~mm} \mathrm{Hg} \text { or PVR } \\
<3 \mathrm{WU}, \mathrm{n}=91)\end{array}$ & 6 & $\begin{array}{l}59 / 75 \% \text { vs } 54^{*} / \\
71 \% \text { in } \mathrm{MPH} \\
\text { and non-MPH, } \\
\text { respectively }\end{array}$ & $\begin{array}{l}\text { Rehospitalisations } \\
\text { and all-cause } \\
\text { mortality }\end{array}$ & NA & $\begin{array}{l}\text { mPAP: } 42 \text { vs } \\
32 \text { in MPH and } \\
\text { non-MPH, } \\
\text { respectively } \\
\text { TPG: } 17 \text { vs } 7 \text {, } \\
\text { respectively }\end{array}$ & 47 & $\begin{array}{l}\text { HR for MPH } \\
0.8(0.59 \text { to } \\
1.08)\end{array}$ & 21 vs 22 & NR & NR & NR & $\begin{array}{l}\text { HR for MPH } 0.89 \\
(0.66 \text { to } 1.20)\end{array}$ \\
\hline $\begin{array}{l}\text { Ghio et al, } \\
2001^{31}\end{array}$ & $\begin{array}{l}\text { RHC with } \mathrm{mPAP} \\
\geq 20 \mathrm{~mm} \mathrm{Hg} \text {, } \\
\text { RV systolic } \\
\text { dysfunction defined } \\
\text { as RVEF }<35 \%\end{array}$ & $\begin{array}{l}377 \text { patients with HF } \\
\text { stratified into: group 1, } \\
\text { normal mPAP/preserved } \\
\text { RVEF ( } n=73) \text {; group 2 } \\
\text { normal mPAP/low RVEF } \\
\text { ( } \mathrm{n}=68 \text { ); group 3, high PAP/ } \\
\text { preserved RVEF ( } \mathrm{n}=21 \text { ); } \\
\text { and group 4, high PAP/low } \\
\text { RVEF ( } \mathrm{n}=215)\end{array}$ & 17.2 & $\begin{array}{l}51 \\
85.7 \%\end{array}$ & $\begin{array}{l}\text { Heart } \\
\text { transplantation and } \\
\text { all-cause mortality }\end{array}$ & NA & 27.9 & 62.3 & NR & NR & NR & NR & $\begin{array}{l}7.3 \text { vs } 12.3 \\
\text { vs } 23.8 \text { vs } 40 \\
\text { in groups } 1, \\
2,3 \text { and } 4,{ }^{*} \\
\text { respectively }\end{array}$ & $\begin{array}{l}\text { HR } 1.1(1.0 \text { to } \\
1.21) \text { per each } \\
5-\mathrm{mm} \mathrm{Hg} \\
\text { increment }\end{array}$ \\
\hline
\end{tabular}




\begin{tabular}{|c|c|c|c|c|c|c|c|c|c|c|c|c|c|c|}
\hline \multirow[b]{2}{*}{$\begin{array}{l}\text { Author, year } \\
\text { published }\end{array}$} & \multirow{2}{*}{$\begin{array}{l}\text { Diagnostic criteria } \\
\text { (RVSP by } \\
\text { echocardiography } \\
\text { or mPAP by } \\
\text { echocardiography } \\
\text { or RHC) }\end{array}$} & \multirow[b]{2}{*}{$\begin{array}{l}\text { Study population (sample } \\
\text { size, heart disease, } \\
\text { NYHA class, type of HF) }\end{array}$} & \multirow[b]{2}{*}{$\begin{array}{l}\text { Mean/ } \\
\text { median } \\
\text { follow-up } \\
\text { (months) }\end{array}$} & \multirow[b]{2}{*}{$\begin{array}{l}\text { Age-years/ } \\
\text { male sex-\% }\end{array}$} & \multirow[b]{2}{*}{$\begin{array}{l}\text { Definition of } \\
\text { outcomes } \\
\text { predicted }\end{array}$} & \multirow{2}{*}{$\begin{array}{l}\text { Proportion } \\
\text { (\%) of } \\
\text { measurable } \\
\text { RVSP }\end{array}$} & \multirow{2}{*}{$\begin{array}{l}\text { Median/mean } \\
\text { ( } \mathrm{mm} \mathrm{Hg} \text { ) } \\
\text { baseline } \\
\text { RVSP (echo) } \\
\text { or mPAP } \\
\text { (RHC) }\end{array}$} & \multirow[b]{2}{*}{$\begin{array}{l}\text { Prevalence } \\
\text { of } \mathrm{PH} \text { at } \\
\text { baseline } \\
\text { (\%) }\end{array}$} & \multirow{2}{*}{$\begin{array}{l}\text { HF } \\
\text { readmission } \\
\text { rate or } \\
\text { adjusted } \\
\text { ORs/HRs } \\
\text { and Cl }\end{array}$} & \multicolumn{4}{|c|}{$\begin{array}{l}\text { Mortality (all-cause) rate at 6, 12, } 24 \text { and } 36 \text { months or } \\
\text { at mean duration of follow-up }\end{array}$} & \multirow{2}{*}{$\begin{array}{l}\text { Adjusted ORs/ } \\
\text { HRs and } \mathrm{Cl} \text { (or } \\
\mathrm{p} \text { value) for } \\
\text { all-cause } \\
\text { mortality, } \\
\text { outcome }\end{array}$} \\
\hline & & & & & & & & & & 6 & 12 & 24 & $\begin{array}{l}\text { mean/ } \\
\text { median } \\
\text { follow-up }\end{array}$ & \\
\hline $\begin{array}{l}\text { Wang et al, } \\
2010^{32}\end{array}$ & RVSP $>30 \mathrm{~mm} \mathrm{Hg}$ & $\begin{array}{l}93 \text { patients with } \mathrm{HF} \\
\text { undergoing cardiac } \\
\text { resynchronisation stratified } \\
\text { into group } 1 \text { (RVSP } \\
>50 \mathrm{mmH}, \mathrm{n}=29) \text {; group } 2 \\
(30<\mathrm{RVSP} \leq 50 \mathrm{~mm} \mathrm{Hg} \text {, } \\
\mathrm{n}=17 \text { ) and group } 3 \text { (RVSP } \\
\leq 30 \mathrm{~mm} \mathrm{Hg}, \mathrm{n}=47 \text { ) }\end{array}$ & $\begin{array}{l}32(6 \text { to } \\
60)\end{array}$ & $\begin{array}{l}59.6 \\
81.7 \%\end{array}$ & $\begin{array}{l}\text { All-cause mortality, } \\
\text { HF mortality }\end{array}$ & NR & NR & 49.5 & NR & $\begin{array}{l}28 \text { vs } 6 \text { vs } \\
17 \% \text { in } \\
\text { groups } 1,2 \\
\text { and } 3, \\
\text { respectively }\end{array}$ & NR & NR & NR & $\begin{array}{l}\text { Non-significant } \\
\text { increased in } \\
\text { all-cause } \\
\text { mortality } \\
(\mathrm{p}=0.33) \text {, } \\
\text { increase in HF } \\
\text { mortality but OR/ } \\
\text { HR not reported }\end{array}$ \\
\hline $\begin{array}{l}\text { Ghio et al, } \\
2013^{33}\end{array}$ & $\begin{array}{l}\text { RVSP }>40 \mathrm{~mm} \mathrm{Hg} \\
\text { and RV dysfunction } \\
\text { defined as TAPSE } \\
<14 \mathrm{~mm}\end{array}$ & $\begin{array}{l}658 \text { patients with chronic } \\
\text { HF stratified into group } 1 \\
\text { (no } \mathrm{PH} \text { no RVD, } \mathrm{n}=256 \text { ), } \\
\text { group } 2 \text { (RVD, no } \mathrm{PH} \text {, } \\
\mathrm{n}=54 \text { ), group } 3 \text { (PH, no } \\
\text { RVD, } \mathrm{n}=167), \text { and group } 4 \\
\text { (RVD and } \mathrm{PH}, \mathrm{n}=67 \text { ) }\end{array}$ & 38 & $\begin{array}{l}63 \\
86 \%\end{array}$ & $\begin{array}{l}\text { All-cause mortality, } \\
\text { urgent cardiac } \\
\text { transplantation or } \\
\text { ventricular fibrillation }\end{array}$ & 83 & 38 & 35.6 & NR & $\begin{array}{l}17.5 \% \text { in } \\
\mathrm{PH} \text { vs } \\
4.5 \% \text { in } \\
\text { non- } \mathrm{PH}\end{array}$ & $\begin{array}{l}21.4 \% \text { in } \mathrm{PH} \\
\text { vs } 8.7 \% \text { in } \\
\text { non- } \mathrm{PH}\end{array}$ & $\begin{array}{l}42.3 \% \text { in } \mathrm{PH} \\
\text { vs } 20.3 \% \text { in } \\
\text { non-PH }\end{array}$ & $\begin{array}{l}59.4 \% \text { in } \mathrm{PH} \\
\text { vs } 45.2 \% \text { in } \\
\text { non-PH }\end{array}$ & $\begin{array}{l}\text { HR } 1.90 \text { ( } 2.18 \text { to } \\
3.06 \text { ) for group } 3 \\
\text { and } 4.27 \text { ( } 3.45 \text { to } \\
7.43 \text { ) for group } 4\end{array}$ \\
\hline \multirow{2}{*}{$\begin{array}{l}\text { Studies in pat } \\
\text { Fawzy et al, } \\
2004^{35}\end{array}$} & ients with heart valve & disease & & & & & & & & & & & & \\
\hline & $\begin{array}{l}\text { Severe PH defined } \\
\text { as RVSP } \\
>50 \mathrm{~mm} \mathrm{Hg}\end{array}$ & $\begin{array}{l}559 \text { patients with MS } \\
\text { undergoing MBV stratified } \\
\text { into three groups: group A } \\
\text { (RVSP <50 mm Hg; } \\
\mathrm{n}=345) \text {; group B (RVSP } \\
50-79 \mathrm{~mm} \mathrm{Hg} \mathrm{n}=183 \text { ) and } \\
\text { group C (RVSP } \\
\geq 80 \mathrm{~mm} \mathrm{Hg} ; \mathrm{n}=31 \text { ) }\end{array}$ & 63.6 & $\begin{array}{l}31 / 28.1 \% \text { vs } \\
30 / 25.1 \% \text { vs } \\
27 / 16.1 \% \text { in } \\
\text { groups A, B } \\
\text { and C, } \\
\text { respectively }\end{array}$ & $\begin{array}{l}\text { Reversibility of PH } \\
\text { following MBV }\end{array}$ & NR & $\begin{array}{l}38.5 \text { vs } 59 \text { vs } \\
97.8 \text { in groups } \\
A, B \text { and } C \text {, } \\
\text { respectively }\end{array}$ & $\begin{array}{l}62 \% \text { vs } 33 \% \\
\text { vs } 5 \% \text { for } \\
\text { groups } \mathrm{A}, \mathrm{B} \text {, } \\
\text { and C, } \\
\text { respectively }\end{array}$ & NR & 0 & 0 & 0 & 0 & $\begin{array}{l}\text { No mortality was } \\
\text { encountered, PH } \\
\text { normalised over } \\
\text { a } 6 \text { to } 12 \text { months }\end{array}$ \\
\hline $\begin{array}{l}\text { Naidoo et al, } \\
1991^{34}\end{array}$ & $\begin{array}{l}\text { RHC with PASP } \\
\geq 30 \mathrm{~mm} \mathrm{Hg}\end{array}$ & $\begin{array}{l}139 \text { patients with AR (69 } \\
\text { undergoing AVS) stratified } \\
\text { into group } 1 \text { (normal or } \\
\text { mild PH) and group 2 } \\
\text { (moderate PH or marked } \\
\text { PH) }\end{array}$ & 6 & $\begin{array}{l}32.9 \text { vs } 36.2 \\
\text { and } 69.7 \text { vs } \\
77.8 \text { in group } 1 \\
\text { and } 2, \\
\text { respectively }\end{array}$ & $\begin{array}{l}\text { Immediate and } \\
6 \text { months } \\
\text { postoperative } \\
\text { mortality }\end{array}$ & NA & $\begin{array}{l}18 \text { vs } 43.7 \text { in } \\
\text { group } 1 \text { and } 2 \text {, } \\
\text { respectively }\end{array}$ & 63.3 & NR & $\begin{array}{l}3 \text { in group } \\
1 \text { vs } 2.8 \% \\
\text { in group } 2\end{array}$ & NR & NR & NR & $\begin{array}{l}\text { No increased in } \\
\text { mortality, HR not } \\
\text { reported }\end{array}$ \\
\hline $\begin{array}{l}\text { Manners } \\
\text { et al, } 1977^{41}\end{array}$ & $\begin{array}{l}\text { RHC with PASP } \\
>70 \mathrm{~mm} \mathrm{Hg}\end{array}$ & $\begin{array}{l}392 \text { patients who had } \\
\text { undergone prosthetic valve } \\
\text { surgery stratified into } 2 \\
\text { PASP }<70 \mathrm{~mm} \mathrm{Hg}, \mathrm{n}=336 \\
\text { or PASP }>70 \mathrm{~mm} \mathrm{Hg} \text {, } \\
\mathrm{n}=56 \text { ) }\end{array}$ & 48 & NR & Hospital mortality & NA & $\begin{array}{l}\text { Mean PASP } \\
\text { was } 93 \mathrm{~mm} \mathrm{Hg}\end{array}$ & NR & NR & NR & NR & NR & $\begin{array}{l}5.4 \% \text { at } \\
4 \text { years in } \\
\text { both } \mathrm{PH} \text { and } \\
\text { non-PH }\end{array}$ & NR \\
\hline $\begin{array}{l}\text { Roseli et al, } \\
2002^{36}\end{array}$ & RVSP $>35 \mathrm{~mm} \mathrm{Hg}$ & $\begin{array}{l}2385 \text { patients undergoing } \\
\text { AVR stratified into } 3 \\
\text { groups: RVSP <35 mm Hg } \\
\text { n=611; RVSP } 35- \\
50 \mathrm{~mm} \mathrm{Hg}, \mathrm{n}=1199 ; \mathrm{RVSP} \\
>50 \mathrm{~mm} \mathrm{Hg}, \mathrm{n}=575\end{array}$ & 51.6 & $\begin{array}{l}74 \\
55 \%\end{array}$ & $\begin{array}{l}\text { All-cause hospital } \\
\text { and late mortality }\end{array}$ & NR & 41 & 74 & NR & $\begin{array}{l}15.8 \text { vs } \\
19.7 \text { vs } \\
25.9\end{array}$ & NR & NR & NR & $\begin{array}{l}\text { Higher RVSP } \\
\text { was predictor of } \\
5 \text { and } 10 \text { years } \\
\text { mortality, HR not } \\
\text { reported }\end{array}$ \\
\hline $\begin{array}{l}\text { Melby et al, } \\
2011^{37}\end{array}$ & RVSP $>35 \mathrm{~mm} \mathrm{Hg}$ & $\begin{array}{l}1080 \text { patients with } A S \\
\text { undergoing AVR, stratified } \\
\text { into } \mathrm{NPH} \text {, (RVSP } \\
<35 \mathrm{~mm} \mathrm{Hg}, \mathrm{n}=574) \text { and } \\
\mathrm{PH} \text { group(mild } \mathrm{PH} \text {, } \\
\text { moderate and severe } \mathrm{PH} \text { ) }\end{array}$ & 48 & $\begin{array}{l}72.3 \text { vs } 70.2 \\
59.1 \text { vs } 57.8 \% \\
\text { in } \mathrm{PH} \text { and non } \\
\mathrm{PH} \text {, } \\
\text { respectively }\end{array}$ & $\begin{array}{l}\text { All-cause operative } \\
\text { and long-term } \\
\text { mortality }\end{array}$ & NR & 51 in $\mathrm{PH}$ group & 46.8 & NR & NR & $\begin{array}{l}17.1 \text { vs } 17.6 \\
\text { vs } 17.1 \text { vs } \\
23.5 \text { for } \\
\text { non-PH, mild, } \\
\text { moderate and } \\
\text { severe } \mathrm{PH}, \\
\text { respectively }\end{array}$ & $\begin{array}{l}25.7 \text { vs } 24 \text { vs } \\
23.2 \text { vs } 32.3\end{array}$ & $\begin{array}{l}25.7 \text { vs } 38.4 \\
\text { vs } 52.7 \text { vs } \\
46.1\end{array}$ & $\begin{array}{l}\text { OR } 1.51 \text { ( } 1.16 \text { to } \\
1.96), \text { persistent } \\
\text { PH after AVR } \\
\text { was associated } \\
\text { with decreased } \\
\text { survival }\end{array}$ \\
\hline $\begin{array}{l}\text { Le Tourneau } \\
\text { et } a l, 2010^{38}\end{array}$ & $\mathrm{RVSP} \geq 50 \mathrm{~mm} \mathrm{Hg}$ & $\begin{array}{l}256 \text { patients with MR } \\
\text { undergoing MVO, stratified } \\
\text { into group } 1 \text { (RVSP } \\
<50 \mathrm{~mm} \mathrm{Hg}, n=174) \text { and } \\
\text { group } 2 \text { (RVSP } \\
\geq 50 \mathrm{~mm} \mathrm{Hg}, n=82)\end{array}$ & 49.2 & $\begin{array}{l}63 \\
66 \%\end{array}$ & $\begin{array}{l}\text { All-cause mortality } \\
\text { Cardiovascular } \\
\text { deaths }\end{array}$ & NR & $45 \pm 14$ & $\begin{array}{l}32 \% \text { had } \\
\text { RVSP } \\
\geq 50 \mathrm{~mm} \mathrm{Hg}\end{array}$ & NR & NR & $\mathrm{NR}$ & $\begin{array}{l}31.6 \text { vs } 31.7 \text { in } \\
\text { groups } 1 \text { and } \\
2 \text {, respectively }\end{array}$ & NR & $\begin{array}{l}\text { HR } 1.43 \text { ( } 1.09 \text { to } \\
1.88) \text { per } \\
10 \mathrm{~mm} \mathrm{Hg} \\
\text { increment of } \\
\text { RVSP }\end{array}$ \\
\hline
\end{tabular}




\begin{tabular}{|c|c|c|c|c|c|c|c|c|c|c|c|c|c|c|}
\hline \multirow[b]{2}{*}{$\begin{array}{l}\text { Author, year } \\
\text { published }\end{array}$} & \multirow{2}{*}{$\begin{array}{l}\text { Diagnostic criteria } \\
\text { (RVSP by } \\
\text { echocardiography } \\
\text { or mPAP by } \\
\text { echocardiography } \\
\text { or RHC) }\end{array}$} & \multirow{2}{*}{$\begin{array}{l}\text { Study population (sample } \\
\text { size, heart disease, } \\
\text { NYHA class, type of HF) }\end{array}$} & \multirow[b]{2}{*}{$\begin{array}{l}\text { Mean/ } \\
\text { median } \\
\text { follow-up } \\
\text { (months) }\end{array}$} & \multirow[b]{2}{*}{$\begin{array}{l}\text { Age-years/ } \\
\text { male sex-\% }\end{array}$} & \multirow[b]{2}{*}{$\begin{array}{l}\text { Definition of } \\
\text { outcomes } \\
\text { predicted }\end{array}$} & \multirow[b]{2}{*}{$\begin{array}{l}\text { Proportion } \\
\text { (\%) of } \\
\text { measurable } \\
\text { RVSP }\end{array}$} & \multirow{2}{*}{$\begin{array}{l}\text { Median/mean } \\
(\mathrm{mm} \mathrm{Hg}) \\
\text { baseline } \\
\text { RVSP (echo) } \\
\text { or mPAP } \\
\text { (RHC) }\end{array}$} & \multirow[b]{2}{*}{$\begin{array}{l}\text { Prevalence } \\
\text { of PH at } \\
\text { baseline } \\
(\%)\end{array}$} & \multirow{2}{*}{$\begin{array}{l}\text { HF } \\
\text { readmission } \\
\text { rate or } \\
\text { adjusted } \\
\text { ORs/HRs } \\
\text { and } \mathrm{Cl}\end{array}$} & \multicolumn{4}{|c|}{$\begin{array}{l}\text { Mortality (all-cause) rate at } 6,12,24 \text { and } 36 \text { months or } \\
\text { at mean duration of follow-up }\end{array}$} & \multirow{2}{*}{$\begin{array}{l}\text { Adjusted ORs/ } \\
\text { HRs and } \mathrm{Cl} \text { (or } \\
\mathrm{p} \text { value) for } \\
\text { all-cause } \\
\text { mortality, } \\
\text { outcome }\end{array}$} \\
\hline & & & & & & & & & & 6 & 12 & 24 & $\begin{array}{l}36 \text { or at } \\
\text { mean/ } \\
\text { median } \\
\text { follow-up }\end{array}$ & \\
\hline $\begin{array}{l}\text { Parker et al, } \\
2010^{7}\end{array}$ & RVSP > 35 mm Hg & $\begin{array}{l}1156 \text { patients with MR or } \\
\text { AR stratified into normal } \\
\text { (RVSP }<30 \mathrm{~mm} \mathrm{Hg}) \text {, } \\
\text { borderline }(31-34 \mathrm{~mm} \mathrm{Hg}) \text {, } \\
\text { mild }(35-40 \mathrm{~mm} \mathrm{Hg} \text { ) or } \\
\text { moderate or greater } \\
(>40 \mathrm{~mm} \mathrm{Hg})\end{array}$ & 87.6 & $\begin{array}{l}72 \\
51 \%\end{array}$ & All-cause mortality & 52 & 29 & NR & NR & NR & NR & NR & NR & $\begin{array}{l}\text { HR for moderate } \\
\text { or greater } \mathrm{PH} \\
1.95(1.58 \text { to } \\
2.41) \text { in } \mathrm{AR} \text { and } \\
1.48(1.26 \text { to } \\
1.75) \text { in } \mathrm{MR}\end{array}$ \\
\hline $\begin{array}{l}\text { Barbieri et al, } \\
2010^{40}\end{array}$ & $\mathrm{RVSP}>50 \mathrm{~mm} \mathrm{Hg}$ & $\begin{array}{l}437 \text { patients with MR, } 35 \% \\
\text { NYHA class III or IV, } \\
\text { normal LVEF, stratified into } \\
\text { NPH (RVSP } \leq 50 \mathrm{~mm} \mathrm{Hg} \text { ) } \\
\text { and PH (RVSP } \\
>50 \mathrm{~mm} \mathrm{Hg} \text { ) }\end{array}$ & 57.6 & $\begin{array}{l}67 \\
66 \%\end{array}$ & $\begin{array}{l}\text { All-cause mortality, } \\
\text { cardiovascular } \\
\text { death, heart failure }\end{array}$ & & 45 & 23 & $\begin{array}{l}1.70(1.10 \text { to } \\
2.62) \text { and } \\
1.19(1.06 \text { to } \\
1.35) \text { for each } \\
10 \mathrm{~mm} \mathrm{Hg} \\
\text { increase of } \\
\text { RVSP }\end{array}$ & NR & & NR & $\begin{array}{l}23 \% \text { at the } \\
\text { mean } \\
\text { follow-up }\end{array}$ & $\begin{array}{l}\text { HR } 2.03(1.30 \text { to } \\
3.18) \text { and } 1.16 \\
\text { (1.03 to } 1.31) \text { for } \\
\text { each } 10 \mathrm{~mm} \mathrm{Hg} \\
\text { increase of } \\
\text { RVSP }\end{array}$ \\
\hline $\begin{array}{l}\text { Kainuma } \\
\text { et al, } 2011^{39}\end{array}$ & $\begin{array}{l}\text { Echocardiography, } \\
\text { PH definition not } \\
\text { specified }\end{array}$ & $\begin{array}{l}46 \text { patients undergoing } \\
\text { MVR, NYHA III or IV, LVEF } \\
<40 \% \text {, stratified into group } \\
1 \text { (RVSP }<40 \mathrm{~mm} \mathrm{Hg} \text {, } \\
n=19 \text { ), group } 2 \text { (moderate } \\
\mathrm{PH}(40<\mathrm{RVSP}<60, \mathrm{n}=17 \text { ) } \\
\text { and group } 3 \text { (RVSP }>60, \\
\mathrm{n}=10 \text { ) }\end{array}$ & 36 & $\begin{array}{l}64 \\
35 \%\end{array}$ & $\begin{array}{l}\text { Cardiac } \\
\text { death, myocardial } \\
\text { infarction, } \\
\text { endocarditis, } \\
\text { thromboembolism, } \\
\text { reoperation } \\
\text { for recurrent MR, } \\
\text { readmission for } \\
\text { heart failure and } \\
\text { fatal arrhythmia }\end{array}$ & NR & 47 & NR & $\begin{array}{l}30 \% \text { in the } \\
\text { severe } \mathrm{PH} \\
\text { but not } \\
\text { significant, } \\
\text { OR and } \mathrm{Cl} \\
\mathrm{NR}\end{array}$ & NR & $\begin{array}{l}15.8 \text { vs } 11.8 \\
\text { vs } 20 \% \text { for } \\
\text { groups } 1,2 \text {, } \\
\text { and } 3, \\
\text { respectively }\end{array}$ & $\begin{array}{l}31.6 \text { vs } 29.4 \\
\text { vs } 30 \%\end{array}$ & $\begin{array}{l}47.4 \text { vs } 82.4 \\
\text { vs } 50 \%\end{array}$ & $\begin{array}{l}\text { HR for all } \\
\text { adverse cardiac } \\
\text { events } 6.9(1.1 \text { to } \\
\text { 44) in group } 3\end{array}$ \\
\hline $\begin{array}{l}\text { Khandhar } \\
\text { et al, } 2009^{43}\end{array}$ & $\begin{array}{l}\text { Severe PH defined } \\
\text { as RVSP } \\
>60 \mathrm{~mm} \mathrm{Hg}\end{array}$ & $\begin{array}{l}506 \text { patients with severe } \\
\text { AR stratified into group } 1 \text {, } \\
\text { severe } \mathrm{PH} \text { with RVSP } \\
>60 \mathrm{~mm} \mathrm{Hg}, \mathrm{n}=83 \text { and } \\
\text { group } 2 \text { (RVSP }<60 \text {, } \\
\mathrm{n}=423) \text {, NYHA NR }\end{array}$ & NR & $\begin{array}{l}63 \\
47 \%\end{array}$ & All-cause mortality & 100 & NR & $\begin{array}{l}16 \% \text { of } \\
\text { severe } \mathrm{PH}\end{array}$ & NR & NR & NR & $\begin{array}{l}21.6 \text { of } \\
\text { patients with } \\
\text { severe } \mathrm{PH}\end{array}$ & NR & $\begin{array}{l}\text { PH was } \\
\text { associated with } \\
\text { increased } \\
\text { mortality in all } \\
\text { groups, OR and } \\
\text { CI NR }\end{array}$ \\
\hline $\begin{array}{l}\text { Malouf et al, } \\
2002^{42}\end{array}$ & $\begin{array}{l}\text { Severe PH defined } \\
\text { as peak TRV } \\
\geq 4 \mathrm{~m} / \mathrm{s}\end{array}$ & $\begin{array}{l}3171 \text { patients with AS of } \\
\text { whom } 47 \text { with severe } \mathrm{PH} \text {, } \\
\text { stratified into group } 1 \text { (no } \\
\text { AVR, } n=10 \text { ) and group } 2 \\
(\mathrm{AVR}, \mathrm{n}=37 \text { ), } 79 \% \text { in NYHA } \\
\text { III and IV }\end{array}$ & 15.3 & $\begin{array}{l}78 \\
47 \%\end{array}$ & All-cause mortality & $\begin{array}{l}63 \% \text { of the } \\
3171 \text { total } \\
\text { population of } \\
\text { patients with } \\
\text { aortic } \\
\text { stenosis }\end{array}$ & $4.16 \mathrm{~m} / \mathrm{s}$ & NA & NR & NR & NR & NR & $\begin{array}{l}80 \% \text { vs } 32 \% \\
\text { in groups } 1 \\
\text { and } 2 \text {, } \\
\text { respectively, } \\
\text { at median } \\
\text { FU }\end{array}$ & $\begin{array}{l}\text { OR for mortality } \\
\text { risk in severe PH } \\
\text { and AVS } 1.76 \\
\text { (0.81 to } 3.35)\end{array}$ \\
\hline $\begin{array}{l}\text { Zuern et al, } \\
2012^{44}\end{array}$ & RVSP >30 mm Hg & $\begin{array}{l}200 \text { patients with AS } \\
\text { undergoing AVR stratified } \\
\text { into } \mathrm{NPH}(\mathrm{RVSP}<30) \text { vs } \\
\text { mild-to-moderate PH ( } 30 \\
<\mathrm{RVSP}<60 \text { ) and severe } \\
\mathrm{PH}(>60 \mathrm{~mm} \mathrm{Hg})\end{array}$ & 31.2 & $\begin{array}{l}72.3 \\
52.5 \%\end{array}$ & All-cause mortality & NR & 36.3 & 61 & NR & NR & $\begin{array}{l}10.2 \text { vs } 14.1 \\
\text { vs } 30.4\end{array}$ & $\begin{array}{l}30.7 \text { vs } 40.4 \\
\text { vs } 60.1\end{array}$ & $\begin{array}{l}2.6,15.2 \\
\text { and } 26.1 \%\end{array}$ & $\begin{array}{l}\text { HR for } \\
\text { mild-to-moderate } \\
\text { PH } 4.9(1.1 \text { to } \\
21.8) \text { and severe } \\
\text { PH } 3.3(0.6 \text { to } \\
\text { 19.7) }\end{array}$ \\
\hline $\begin{array}{l}\text { Ben-Dor } \\
\text { et al, } 2011^{45}\end{array}$ & $\mathrm{RVSP}>40 \mathrm{~mm} \mathrm{Hg}$ & $\begin{array}{l}509 \text { patients with AS } \\
\text { divided into group } 1 \text { (RVSP } \\
<40 \mathrm{~mm} \mathrm{Hg}, n=161 \text { ); group } \\
2 \text { (RVSP } 40-59, n=175 \text { ) } \\
\text { and group } 3 \text { (RVSP } \\
>60 \mathrm{~mm} \mathrm{Hg}, n=173 \text { ) }\end{array}$ & 6.73 & $\begin{array}{l}82.3 \text { vs } 82.4 \text { vs } \\
80.5 \text { in groups } \\
1,2 \text { and } 3, \\
\text { respectively, } \\
>75 \%\end{array}$ & All-cause mortality & NR & $\begin{array}{l}33.7 \text { vs } 49.3 \text { vs } \\
70.7 \text { in groups } \\
1,2, \text { and } 3 \\
\text { respectively }\end{array}$ & 68.3 & NR & NR & NR & NR & $\begin{array}{l}21.7 \text { vs } 39.3 \\
\text { vs } 49.1 \text { in } \\
\text { groups } 1,2 \\
\text { and } 3, \\
\text { respectively } \\
\text { at median } \\
\mathrm{FU}^{*}\end{array}$ & $\begin{array}{l}\text { PH was } \\
\text { significantly } \\
\text { associated with } \\
\text { increase in } \\
\text { mortality, OR/HR } \\
\text { not reported }\end{array}$ \\
\hline $\begin{array}{l}\text { Yang et al, } \\
2012^{46}\end{array}$ & RVSP $>40 \mathrm{~mm} \mathrm{Hg}$ & $\begin{array}{l}845 \text { patients who } \\
\text { underwent valve surgery } \\
\text { and/or CABG ( } 444 \text { without } \\
\text { PH or NPH vs } 401 \mathrm{PH} \text { ), all } \\
\text { with LVEF }<40 \%\end{array}$ & 39 & $\begin{array}{l}65.2 \text { vs } 67.8 \\
78.8 \text { vs } 72.6 \% \\
\text { in NPH and PH } \\
\text { group, } \\
\text { respectively }\end{array}$ & $\begin{array}{l}\text { Postoperative } \\
\text { complications and } \\
\text { mortality }\end{array}$ & & NR & NR & NR & NR & $\begin{array}{l}4.6 \text { vs } 13.9 \text { in } \\
\mathrm{NPH} \text { vs } \mathrm{PH} \\
\text { group, } \\
\text { respectively }\end{array}$ & NR & $\begin{array}{l}16.7 \text { vs } \\
30.6^{*} \text { in } \\
\text { NPH vs PH } \\
\text { group, } \\
\text { respectively }\end{array}$ & $\begin{array}{l}\text { OR for mild/ } \\
\text { moderate } \mathrm{PH} \\
1.475(1.119 \text { to } \\
1.943)\end{array}$ \\
\hline
\end{tabular}


Table 2 Continued

\begin{tabular}{|c|c|c|c|c|c|c|c|c|c|c|c|c|c|c|}
\hline \multirow[b]{2}{*}{$\begin{array}{l}\text { Author, year } \\
\text { published }\end{array}$} & \multirow{2}{*}{$\begin{array}{l}\text { Diagnostic criteria } \\
\text { (RVSP by } \\
\text { echocardiography } \\
\text { or mPAP by } \\
\text { echocardiography } \\
\text { or RHC) }\end{array}$} & \multirow{2}{*}{$\begin{array}{l}\text { Study population (sample } \\
\text { size, heart disease, } \\
\text { NYHA class, type of HF) }\end{array}$} & \multirow[b]{2}{*}{$\begin{array}{l}\text { Mean/ } \\
\text { median } \\
\text { follow-up } \\
\text { (months) }\end{array}$} & \multirow[b]{2}{*}{$\begin{array}{l}\text { Age-years/ } \\
\text { male sex-\% }\end{array}$} & \multirow[b]{2}{*}{$\begin{array}{l}\text { Definition of } \\
\text { outcomes } \\
\text { predicted }\end{array}$} & \multirow[b]{2}{*}{$\begin{array}{l}\text { Proportion } \\
\text { (\%) of } \\
\text { measurable } \\
\text { RVSP }\end{array}$} & \multirow{2}{*}{$\begin{array}{l}\text { Median/mean } \\
(\mathrm{mm} \mathrm{Hg}) \\
\text { baseline } \\
\text { RVSP (echo) } \\
\text { or mPAP } \\
\text { (RHC) }\end{array}$} & \multirow[b]{2}{*}{$\begin{array}{l}\text { Prevalence } \\
\text { of } \mathrm{PH} \text { at } \\
\text { baseline } \\
(\%)\end{array}$} & \multirow{2}{*}{$\begin{array}{l}\text { HF } \\
\text { readmission } \\
\text { rate or } \\
\text { adjusted } \\
\text { ORs/HRs } \\
\text { and Cl }\end{array}$} & \multicolumn{4}{|c|}{$\begin{array}{l}\text { Mortality (all-cause) rate at 6, 12, } 24 \text { and } 36 \text { months or } \\
\text { at mean duration of follow-up }\end{array}$} & \multirow{2}{*}{$\begin{array}{l}\text { Adjusted ORs/ } \\
\text { HRs and } \mathrm{Cl} \text { (or } \\
\mathrm{p} \text { value) for } \\
\text { all-cause } \\
\text { mortality, } \\
\text { outcome }\end{array}$} \\
\hline & & & & & & & & & & 6 & 12 & 24 & $\begin{array}{l}36 \text { or at } \\
\text { mean/ } \\
\text { median } \\
\text { follow-up }\end{array}$ & \\
\hline $\begin{array}{l}\text { Nozohoor } \\
\text { et } a l, 2012^{47}\end{array}$ & RVSP $>50 \mathrm{~mm} \mathrm{Hg}$ & $\begin{array}{l}270 \text { patients with MR } \\
\text { undergoing MVS, stratified } \\
\text { into } \mathrm{NPH} \text { group (RVSP } \\
<50 \mathrm{~mm} \mathrm{Hg} \text { ) and } \mathrm{PH} \text { group } \\
(\mathrm{RVSP} \geq 50 \mathrm{~mm} \mathrm{Hg} \text { ) }\end{array}$ & 61.2 & $\begin{array}{l}61.5 \text { vs } 66.5 \\
70 \text { vs } 54 \% \text { in } \\
\text { no } \mathrm{PH} \text { and } \mathrm{PH} \\
\text { group, } \\
\text { respectively }\end{array}$ & $\begin{array}{l}\text { Perioperative } \\
\text { complications and } \\
\text { all-cause late } \\
\text { mortality }\end{array}$ & NR & NR & 27 & NR & NR & $\begin{array}{l}7.6 \text { vs } 8.2 \text { in } \\
\text { no } \mathrm{PH} \text { and } \\
\mathrm{PH} \text {, } \\
\text { respectively }\end{array}$ & $\begin{array}{l}22.4 \text { vs } 17.6 \text { in } \\
\text { no } \mathrm{PH} \text { and } \mathrm{PH}, \\
\text { respectively }\end{array}$ & $\begin{array}{l}31.1 \text { in both } \\
\text { groups }\end{array}$ & $\begin{array}{l}\text { HR } 4.3 \text { (1.1 to } \\
\text { 17.4) during the } \\
\text { initial 3 years } \\
\text { after MVS }\end{array}$ \\
\hline $\begin{array}{l}\text { Ward and } \\
\text { Hancock } \\
1975^{48}\end{array}$ & $\begin{array}{l}\text { RHC with extreme } \\
\text { PH defined as } \\
\text { SPAP > }>00 \mathrm{~mm} \mathrm{Hg} \\
\text { and PVR }>10 \text { WU: } \\
8.2 \%\end{array}$ & $\begin{array}{l}\text { Mitral valve disease } \\
\text { (n=586), } 48 \text { extreme PH } \\
\text { stratified into group } 1 \text { (no } \\
\text { operation), group } 2 \text { (all } \\
\text { surgical) and group } 3 \\
\text { (survive after surgery) }\end{array}$ & 69.6 & $\begin{array}{l}46.2 \text { vs } 42.4 \\
43 \text { vs } 29 \% \text { in } \\
\text { group } 1 \text { and } 2 \\
\text { respectively }\end{array}$ & All-cause mortality & NA & 105 vs 96.6 & 8.2 & NA & NR & NR & NR & NR & $\begin{array}{l}\text { Extreme PH was } \\
\text { associated with } \\
\text { higher mortality, } \\
\text { and surgery } \\
\text { improved survival }\end{array}$ \\
\hline $\begin{array}{l}\text { Ghoreishi } \\
\text { et } a l, 2012^{49}\end{array}$ & $\begin{array}{l}\mathrm{sPAP}>40 \mathrm{~mm} \mathrm{Hg} \\
\text { using RHC in } 591 \\
\text { patients and RVSP } \\
>40 \mathrm{~mm} \mathrm{Hg} \text { using } \\
\mathrm{DE}\end{array}$ & $\begin{array}{l}873 \text { patients with MR who } \\
\text { underwent MVS, stratified } \\
\text { into NPH and PH group } \\
\text { (mild, moderate, severe) } \\
\text { NHYA not reported }\end{array}$ & 35 & $\begin{array}{l}59 \\
59 \%\end{array}$ & $\begin{array}{l}\text { Hospital mortality, } \\
\text { Late all-cause } \\
\text { mortality }\end{array}$ & NR & $\begin{array}{l}46 \text { (echo), and } \\
\text { sPAP was } 43 \\
\text { by RHC }\end{array}$ & 53 & NR & NR & $\begin{array}{l}16.2 \text { in non } \\
\mathrm{PH} \text { vs } 32 \% \text { in } \\
\mathrm{PH} \text { group* }\end{array}$ & $\begin{array}{l}33.9 \text { in non } \mathrm{PH} \\
\text { vs } 48.1 \% \text { in } \\
\text { PH group* }\end{array}$ & $\begin{array}{l}51.8 \text { in non } \\
\mathrm{PH} \text { vs } \\
60.9 \% \text { in } \mathrm{PH} \\
\text { group }^{\star}\end{array}$ & $\begin{array}{l}\text { HR } 1.018 \text { ( } 1.007 \\
\text { to } 1.028) \text { per } \\
\text { each } 1 \mathrm{~mm} \mathrm{Hg} \\
\text { increment in } \\
\text { RVSP }\end{array}$ \\
\hline $\begin{array}{l}\text { Cam A et al, } \\
2011^{50}\end{array}$ & $\begin{array}{l}\text { RHC with severe } \\
\text { PH defined as } \\
\mathrm{mPAP}>35 \mathrm{~mm} \mathrm{Hg}\end{array}$ & $\begin{array}{l}317 \text { patients with AS, } 35 \\
\text { with severe PH underwent } \\
\text { surgery and were } \\
\text { compared to } 114 \text { mild } \\
\text { moderate } \mathrm{PH} \text { and to } 46 \\
\text { severe } \mathrm{PH} \text { treated } \\
\text { conservatively, NHYA not } \\
\text { reported }\end{array}$ & 11.3 & $\begin{array}{l}71 / 53.5 \\
\text { (mild-moderate } \\
\text { PH) vs } 75 / 51.4 \\
\text { (severe PH) }\end{array}$ & All-cause mortality & NA & $\begin{array}{l}22.5 \\
\text { (mild-moderate } \\
\text { PH) vs } 45.3 \\
\text { (severe PH) }\end{array}$ & 47.0 & NR & NR & NR & NR & 74.5 vs 75.5 & $\begin{array}{l}\text { HR } 1.008 \text { ( } 0.9 \text { to } \\
1.11) \text { and early } \\
\text { postoperative } \\
\text { reduction in } \\
\text { mPAP } 0.93(1.2 \\
\text { to } 12.5)\end{array}$ \\
\hline $\begin{array}{l}\text { Pai et al, } \\
2007^{51}\end{array}$ & $\begin{array}{l}\text { Severe PH defined } \\
\text { as RVSP } \\
>60 \mathrm{~mm} \mathrm{Hg}\end{array}$ & $\begin{array}{l}116 \text { patients (of } 740 \text { severe } \\
\text { AS) with severe PH among } \\
\text { which } 36 \text { underwent AVR } \\
\text { and were compare to } 83 \\
\text { remaining }\end{array}$ & 18 & $\begin{array}{l}75 \\
39 \%\end{array}$ & All-cause mortality & NR & 69 & $\begin{array}{l}15.7 \% \\
\text { (severe } \mathrm{PH})\end{array}$ & NR & NR & NR & $\begin{array}{l}30.5(\mathrm{PH}) \text { vs } \\
15.5(\mathrm{NPH})\end{array}$ & NR & $\begin{array}{l}\text { AVR benefit HR } \\
0.28(0.16 \text { to } \\
0.51) \\
\text { independent of } \\
\mathrm{PH}\end{array}$ \\
\hline
\end{tabular}


Figure 1 Flow diagram of literature search process. LHD, left heart disease; $\mathrm{PH}$, pulmonary hypertension.

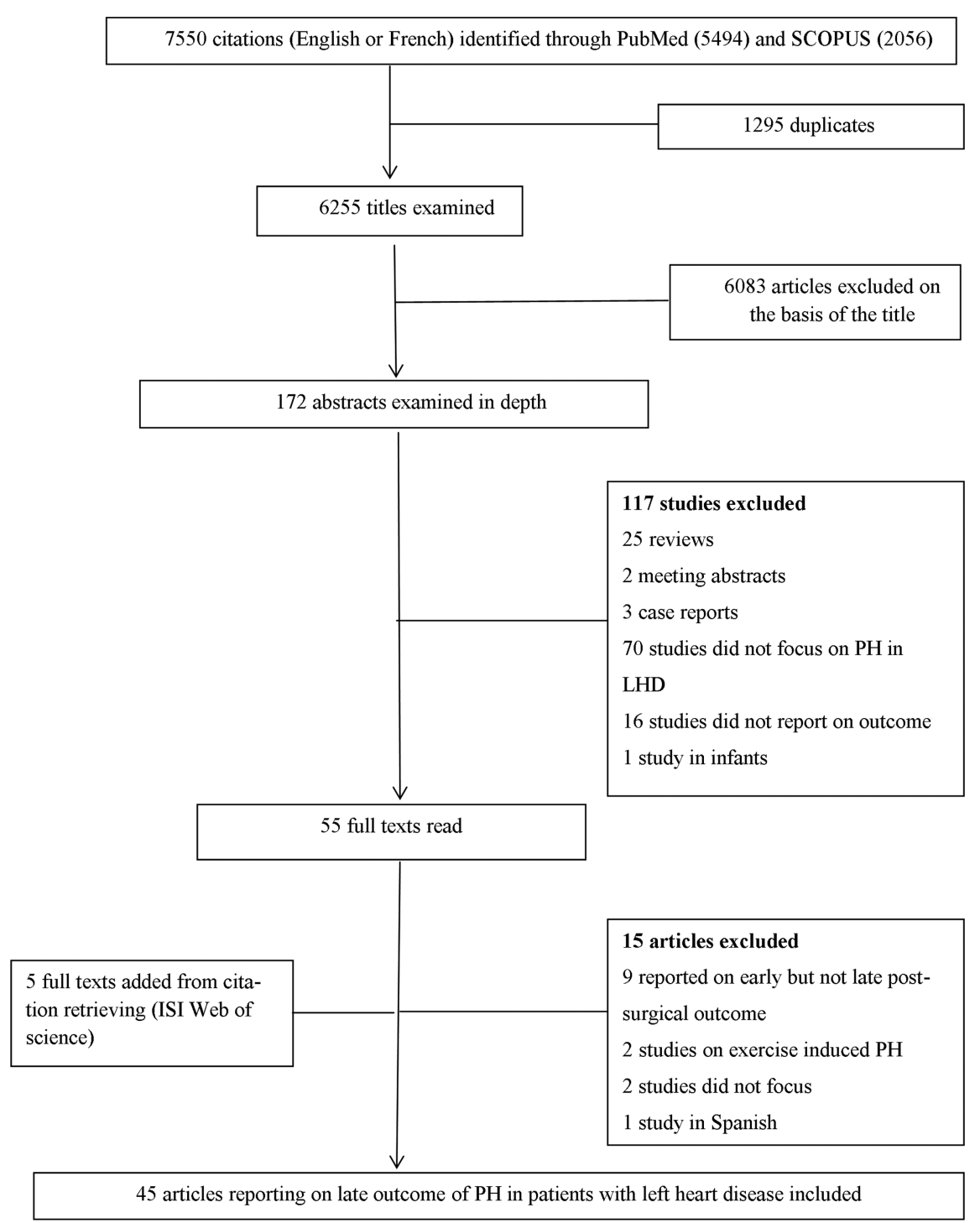

screened by full text of which 15 were excluded for various reasons (figure 1). Five studies were identified via citation search. Therefore, 45 articles were included in the final review among which $86.7 \%$ were published between 2003 and 2013 (see online supplementary figure $\mathrm{S} 1$ ).

\section{Study characteristics and methodological quality}

The characteristics and methodological quality of the 45 included studies are described in table 1 . The overall quality score ranged from 29.5 to 72.5 points with a median of 63.5. Based on the cut-offs of $\geq 60$ and $\geq 45$ points, respectively, we classified 34 articles as being of high quality, 7 as moderate-to-high quality and four as low-quality studies (table 1). Studies of high quality were recent and scored well on patient selection, outcome measurement, statistical analysis and presentation. Studies classified as moderate/low quality scored relatively well on patient selection, but poorly on study attrition, statistical analysis and presentation. Twenty-four $(53.3 \%)$ studies were from the USA, 12 (26.6\%) from
Europe (four from UK, three from Italy and one each from Spain, Germany, Denmark, France and Sweden), $6(13.3 \%)$ from Asia (two from Japan, one each from India, China, Korea and Australia) and 1 from South Africa. One study was multicentric across Europe and the $\mathrm{USA}^{40}$ and another one was multicentric across the USA and Canada. ${ }^{30}$ Only three population-based cohorts were reported including two prospective ${ }^{13} \quad 29$ and one retrospective study. ${ }^{14}$ For the remaining 42 hospital-based cohort studies, 20 had a retrospective design. The number of participants ranged from 46 to 2385 in hospital-based and from 244 to 1049 in population-based studies. The proportion of men ranged from $21 \%$ to $91 \%$, and mean/median age from 63 to 82 years. Twenty-six studies were in patients with heart failure (HF) and cardiomyopathies (two in heart failure with preserved ejection fraction ( $\mathrm{HFpEF})$ ) and 19 in patients with valve disease.

Twelve studies defined PH using RHC and 32 studies using DE. One study defined PH using both RHC and DE. Studies applied variable definitions of $\mathrm{PH}$ using both 
RHC (based on mPAP $>25$ or $30 \mathrm{~mm} \mathrm{Hg}$, or on systolic pulmonary artery pressure (sPAP) $>50 \mathrm{~mm} \mathrm{Hg}$, or sPAP $>40 \mathrm{~mm} \mathrm{Hg}$, or on pulmonary vascular resistance (PVR) $>2.5$ wood units (WU)) and DE (based on RVSP with cut-offs varying from 35 to $50 \mathrm{~mm} \mathrm{Hg}$, or based on a mPAP $>25 \mathrm{~mm} \mathrm{Hg}^{8}$ or on a right ventricular tricuspid gradient (RVTG) $>25 \mathrm{~mm} \mathrm{Hg}$ ). ${ }^{25}$ Prevalence of $\mathrm{PH}$ in $\mathrm{HF}$ ranged from $22 \%$ to $83.3 \%$ overall, $22-83.3 \%$ in studies of PH based on DE and 23-76\% in studies of PH based on RHC (see online supplementary figure S2).

\section{Outcome of PH}

\section{Admissions for heart failure}

The duration of follow-up ranged from 6 to 87.6 months overall, 6-69.6 months in studies of PH based on RHC definition and 6-87.6 months in studies of $\mathrm{PH}$ based on DE definition. Readmission rates, when reported, ranged from $9.2 \%$ to $75 \%$ overall and $9.2-75 \%$ in studies of PH based on DE definition. Only one study with PH definition based on RHC reported a readmission rate of $27 \%$ (table 2). Admissions or readmissions for $\mathrm{HF}$ were reported in nine studies all based on DE definition among which seven reported HRs or ORs for admission/readmission in relation with PH. Effect estimates for six of the seven studies were statistically significant.

\section{Mortality}

Mortality was reported in all studies (table 2); however, not all studies provided multivariable-adjusted effect estimates of mortality risk associated with $\mathrm{PH}$. $\mathrm{PH}$ was associated with increased all-cause mortality in 24 of 26 studies of HF, among which 6 studies were of $\mathrm{PH}$ based on RHC definition, while two studies failed to report an association between $\mathrm{PH}$ and all-cause mortality at 6 months. Of these two studies, one used PH definition based on RHC and was a multicentric trial of HF that reported effect estimates for mortality risk from $\mathrm{PH}$ $(\mathrm{HR}=0.89 \quad(95 \%$ CI 0.66 to 1.20$)) ;{ }^{30}$ while the other one $^{32}$ did not. When reported, mortality rates at 12 months ranged from $0 \%$ to $32 \%$ overall, $0 \%$ to $32 \%$ in studies of $\mathrm{PH}$ based on $\mathrm{DE}$ and $2.9 \%$ to $18 \%$ in studies of PH based on RHC (see online supplementary figure S3). As summarised in table 3, over 35 potential predictors of mortality were tested across studies with variable and often inconsistent effects on the outcome of interest. Age was associated with mortality in 14 studies (among which 11 studies of $\mathrm{PH}$ were based on $\mathrm{DE}$ ), male gender in $3 / 11$ studies (all based on DE), LVEF in 6/10 studies, right ventricular (RV) function in $3 / 3$ studies and renal disease (rising creatinine, decreasing glomerular filtration rate (GFR) or dialysis) in 6/17 studies (all based on DE), functional class (New York Heart Association (NYHA) or WHO) in 7/12 studies (five based on DE) while the 6 min walking distance was tested in only one study but was not integrated in the multivariable analysis for outcome risk. ${ }^{32}$

\section{DISCUSSION}

An increasing number of studies have assessed the risk of readmission and mortality in patients with LHD-related PH over the last decade, and mostly in North America and Europe. Available studies are mostly consistent on the adverse effect of PH (whether assessed using DE or RHC) on mortality risk in patients with heart failure as well as those with mitral valve disease, but less unanimous in those with aortic valve disease. The consistent adverse effect of $\mathrm{PH}$ in this population highlights the importance of early diagnosis of $\mathrm{PH}$ to reduce mortality. While available studies have been overall of acceptable quality, substantial heterogeneity in the study population, PH definition and measurement, outcome definitions as well as other prognostic factors limit direct comparisons across studies. Information on readmission for heart failure was limited and the assessment of other prognostic factors in an integrated multivariable model was very heterogeneous.

\section{Mortality in patients with PH and heart failure with reduced ejection fraction}

While PH was an independent prognostic factor for mortality in fatal-outcome studies, the prevalence of $\mathrm{PH}$ and effects on mortality varied according to LVEF. Differences in the prevalence of $\mathrm{PH}$ could be explained at least in part by population heterogeneity (age, level of HF, HF centres or community study) and differences in the criteria used to define $\mathrm{PH}$ across studies with a variety of cut-off values. Regardless of the prevalence of PH in HFrEF, there seems to be no uniformity in the association between the magnitude of reduction in LVEF, and the presence or absence of $\mathrm{PH}$ and the effects of $\mathrm{PH}$ on mortality risk. It is possible that the small size of studies and the short duration of follow-up precluded the accumulation of a substantial number of events to allow the detection of a relationship, if any. Furthermore, although the precise haemodynamic threshold beyond which RVSP is invariably associated with mortality is subject to debate; the risk of death associated with PH seems to increase with higher RVSP. ${ }^{6} 121316$ A possible pathophysiological explanation is that early and higher vascular remodelling occurs in patients with HF and severe $\mathrm{PH}$, causing a reactive or 'postcapillary $\mathrm{PH}$ with a precapillary component', which in turn has a greater impact on the RV function. Equally, RV systolic function has been shown to be highly influenced by pressure overload and by vascular resistance in the pulmonary region ${ }^{50}$; and RV function assessed using RHC or echocardiography has been shown to be associated with mortality. ${ }^{30} 3133$ It is, however, remarkable that one study ${ }^{30}$ reported no interaction between $\mathrm{PH}$ and RV function, with both variables being independently associated with mortality. This highlights the fact that RV function in HF does not only depend on pulmonary pressure but may also reflect intrinsic myocardial disease. As suggested by Vachiery et at there might be a spectrum of clinical phenotypes of RV failing in PH-LHD that might evolve from one to the other, from isolated postcapillary $\mathrm{PH}$ with little effect on 
Table 3 Other prognostic factors associated with mortality in patients with pulmonary hypertension associated with left heart disease

\begin{tabular}{|c|c|c|c|c|}
\hline \multirow[b]{2}{*}{ Factor } & \multicolumn{2}{|c|}{$\begin{array}{l}\text { Number of studies } \\
\text { reporting }\end{array}$} & \multicolumn{2}{|c|}{$\begin{array}{l}\text { Number of studies in which } \\
\text { the factor was associated with } \\
\text { poor outcome }\end{array}$} \\
\hline & overall & $\begin{array}{l}\text { Studies based } \\
\text { on } D E\end{array}$ & $\begin{array}{l}\text { Studies of PH } \\
\text { based on DE }\end{array}$ & $\begin{array}{l}\text { Studies of PH } \\
\text { based on RHC }\end{array}$ \\
\hline Age & 14 & 11 & 11 & 3 \\
\hline Sex (male vs female) & 11 & 9 & 3 & 0 \\
\hline Racial/ethnic group & 2 & 2 & 0 & 0 \\
\hline HF episodes & 5 & 5 & 2 & 0 \\
\hline Prior hypertension & 5 & 5 & 1 & 0 \\
\hline History of diabetes & 8 & 8 & 3 & 0 \\
\hline Smoking & 3 & 3 & 0 & 0 \\
\hline History of cardiovascular disease & 1 & 1 & 1 & 0 \\
\hline Functional class (NYHA/WHO) & 12 & 9 & 5 & 2 \\
\hline Killip class for MI & 2 & 2 & 2 & 0 \\
\hline Heart rate & 2 & 2 & 0 & 0 \\
\hline Systolic BP & 4 & 4 & 2 & 0 \\
\hline Diastolic BP & 1 & 1 & 1 & 0 \\
\hline Mean BP & 1 & 1 & 1 & 0 \\
\hline $\mathrm{SPO}_{2}$ & 3 & 3 & 1 & 0 \\
\hline Hypotension & 1 & 1 & 1 & 0 \\
\hline Atrial fibrillation & 5 & 5 & 5 & 0 \\
\hline Ischaemic aetiology of HF & 4 & 4 & 0 & 0 \\
\hline Urea & 2 & 2 & 1 & 0 \\
\hline Kidney disease (by creatinine, GFR or haemodialysis) & 17 & 14 & 6 & 0 \\
\hline BNP & 3 & 3 & 2 & 0 \\
\hline Haemoglobin & 2 & 2 & 0 & 0 \\
\hline Presence of COPD & 4 & 3 & 3 & 0 \\
\hline $\begin{array}{l}\text { Use of medications (ACEI and or beta blockers or } \\
\text { spironolactone) }\end{array}$ & 6 & 6 & 3 & 0 \\
\hline LVEF & 10 & 10 & 6 & NA \\
\hline LV end-diastolic diameter/index & 6 & 6 & 3 & NA \\
\hline Atrial diameter & 1 & 1 & 1 & NA \\
\hline Deceleration time & 1 & 1 & 0 & NA \\
\hline RV function (by TAPSE or other means) & 3 & 3 & 3 & NA \\
\hline Functional mitral regurgitation & 5 & 5 & 4 & NA \\
\hline RVSP $\geq 50$ or $>60 \mathrm{~mm} \mathrm{Hg}$ & 9 & 9 & 5 & NA \\
\hline End diastolic pulmonary regurgitation & 1 & 1 & 1 & NA \\
\hline
\end{tabular}

the RV to more advanced disease where the failing RV is the key determinant of outcome.

\section{Mortality in patients with $\mathrm{PH}$ and heart failure with preserved ejection fraction}

Over the past decades, the increasing prevalence of $\mathrm{HFpEF}^{51}$ has been paralleled by an increasing presence of $\mathrm{PH}$ in patients with $\mathrm{HFpEF}^{5}{ }^{6}$ When compared to heart failure with reduced ejection fraction (HFrEF), patients with HFpEF have their subset of risk factors; but finally, $\mathrm{PH}$ conveys similar morbidity and mortality risk in the two subgroups of patients. ${ }^{13}{ }^{17}$ The current incomplete understanding of HFpEF limits our ability to explain why these patients develop PH. However, it is estimated that over time left atrium and ventricular filling pressure from compromised left ventricle and, in some, left atrium relaxation and distensibility can lead to elevated pulmonary venous pressure, triggering vasoconstriction and arterial remodelling. ${ }^{4}{ }^{5}$ In total, the finding of $\mathrm{PH}$ as an independent prognostic factor for mortality in patients with HF tends to support the suggestion that $\mathrm{PH}$ should be considered as a potential therapeutic target at least in the group of patients with $\mathrm{HF}$ who exhibit persisting $\mathrm{PH}$ after optimisation of $\mathrm{HF}$ therapy. In this line, targeting both pulmonary vasculature and the heart would probably be more beneficial.

\section{Mortality in patients with $\mathrm{PH}$ related to valvular heart disease}

$\mathrm{PH}$ due to valvular heart disease (VHD) was not always related to mortality risk, ${ }^{38} 3945$ which is in contrast with $\mathrm{PH}$ in patients with heart failure. A simple explanation 
of this difference could be that the prevalence and severity of $\mathrm{PH}$ correlates with the severity and type of VHD. Although mitral stenosis (MS) has been the classical disease associated with $\mathrm{PH}-\mathrm{LHD}$ and reactive $\mathrm{PH}$ was initially described in these patients ${ }^{4}$; it is, however, noticeable that PH due to MS has received little attention over the last decade, probably because of the progressive decline in RHD in Western countries. Interestingly, the two studies included showed that surgery was safe and improved survival in patients with $\mathrm{PH}$ due to $\mathrm{MS}^{35}{ }^{48}$ with $\mathrm{PH}$ regressing to normal levels over 6-12 months after successful Mitral Balloon Valvotomy (MBV). ${ }^{35}$ In mitral regurgitation (MR), nearly all cohort studies on outcomes of severe $\mathrm{PH}$ reported increased mortality. ${ }^{38} 39404649$ The relevance of this finding is that $\mathrm{PH}$ can serve both as an indication for proceeding to surgical or catheter-based interventions, and also as an operative risk factor for mitral valve interventions. ${ }^{54}$ By contrast, $\mathrm{PH}$ is not as common in the aortic valve surgical cohort. Mortality rates in different studies of patients with VHD depends on comorbidities, exclusion criteria and definition for PH. Studies that also evaluated changes in $\mathrm{PH}$ following valve surgery showed a decline in pulmonary pressures following surgery. ${ }^{35} 455055$ It is worth noting that the pathophysiology of the pulmonary vasculature in PH due to VHD is similar to that in patients with $\mathrm{HF}^{1}$

\section{Hospitalisations and other prognostic factors}

The paucity of information on the effect of PH-LHD on hospitalisations or rehospitalisations as has been shown in this study highlights the need for more evidence on this outcome. Such information is important to fully characterise and quantify the contribution of PH-LHD to the global burden of disease, and assess future improvement from treating the underlying LHD and/or controlling $\mathrm{PH}$ in patients with LHD.

Of the 35 other potential prognostic factors of mortality in patients with $\mathrm{PH}$ that were tested in multivariable models across studies, investigations on echocardiographic parameters suggested that $\mathrm{PH}>60 \mathrm{~mm} \mathrm{Hg}$ was associated with worse mortality in seven of the nine studies. Similarly, a greater degree of MR, deceleration time when reported ${ }^{26}$ and RV function were almost constantly associated with adverse outcome while LVEF was associated with adverse outcome in 6 of the 10 studies. In the evolution of LHD, RV dysfunction usually occurs as a turning point. It shall be noted that $\mathrm{PH}$ incorporates information on diastolic function, MR and pulmonary vascular disease, and this might explain the pivotal role of $\mathrm{PH}$ in gauging the prognosis of patients with HF.

\section{Strengths and limitations of the studies included in the review}

The first limitation of the studies included in our review is the possibility of study population bias. The majority of studies originated from Western countries and included predominantly Caucasians and reported mostly on PH-LHD in a population with high prevalence of ischaemic heart disease. This precludes the generalisability of our findings to developing countries where aetiologies of LHDs are less of ischaemic origin and are more dominated by systemic hypertension, dilated cardiomyopathies and RHD in a younger population. ${ }^{56}$ Therefore, PH-LHD may have a different prognosis in developing countries. Second, studies included in this review were defined $\mathrm{PH}$ based either on DE or RHC. RHC remains the gold standard to diagnose and confirm $\mathrm{PH}$, but performing RHC on all patients with dyspnoea would bear excessive risks and be impractical in resource-limited settings. DE on the other hand is widely available, safe and relatively cheap for diagnosing $\mathrm{PH}$, although the reproducibility of the approach in some circumstances has been questioned. However, a systematic review on the diagnostic accuracy of $\mathrm{DE}$ in $\mathrm{PH}$ by Janda et $a \tilde{l}^{\tilde{7}}$ has shown that the correlation of pulmonary artery systolic pressure by DE compared to RHC was good with a pooled correlation coefficient of 0.70 (95\% CI 0.67 to 0.73 ). However, studies to date examining the prognostic impact of PH in LHD have been performed in heterogeneous populations, using variable definitions of $\mathrm{PH}$ based both on RHC and echocardiography parameters, thus limiting any possibility of pooling. Finally, readmissions were not frequently reported and multivariable analysis when performed was characterised by a great heterogeneity in the number and range of candidate predictors included in the models, thus limiting interpretation and generalisability. Therefore, findings on these other prognostic factors must be interpreted with caution. For studies that performed only univariate analysis, we cannot rule out the possibility that the reported factors may not preserve a significant association with the outcome once adjusted for the effect of other extraneous factors. In spite of these limitations, the majority of studies included were recent and all reported on the relation of PH-LHD with all-cause mortality, making the conclusions on this relation appropriate for contemporary Western populations.

\section{Strengths and limitations of the review}

First, by restricting our search strategy to full-report articles published in English and French, and in journals available in the used electronic databases, we cannot rule out the possibility of language or publication bias. Second, we used the QUIPS instrument, designed for prognosis studies, to address common sources of bias. The QUIPS, however, lacks discriminative power; we addressed this by using the scoring algorithm suggested by de Jonge et al. ${ }^{6}$ This scoring algorithm can still be subject to criticisms, especially because the cut-off points used to determine the quality of the studies are quite arbitrary. Third, because of important heterogeneity in the included studies, we were not able to pool data to perform a meta-analysis or to stratify data by clinically important subgroups (such as mild, moderate or severe $\mathrm{PH})$. However, to the best of our knowledge, this is the 
first systematic review on determinants of hospitalisations and mortality in patients with PH-LHD, and the search strategy used allowed us to present the results of several recent and high-quality publications on the topic.

\section{CONCLUSION}

The majority of studies included in this review showed that $\mathrm{PH}$ is an independent predictor of mortality in patients with LHD, with the more consistent evidence being in those with $\mathrm{HF}$ and MR. Information on readmission for heart failure was somehow very limited. The majority of this information derives from studies in Western and developed countries, and may not apply to populations in other settings. All together, these findings suggest that the hypothesis of targeting $\mathrm{PH}$ to improve the outcomes of patients with LHD s should be actively investigated.

\section{Author affiliations}

${ }^{1}$ Douala General Hospital and Buea Faculty of Health Sciences, Douala, Cameroon

${ }^{2}$ Department of Medicine, Faculty of Health Sciences, University of Cape Town, Cape Town, South Africa

${ }^{3}$ Non-Communicable Diseases Research Unit, South African Medical Research Council, Cape Town, South Africa

${ }^{4}$ Faculty of Health Sciences, Clinical Infectious Diseases Research Initiative, Institute of Infectious Diseases and Molecular Medicine, University of Cape Town, Cape Town, South Africa

${ }^{5}$ Division of Infectious Diseases and HIV Medicine, Department of Medicine, University of Cape Town, Cape Town, South Africa

${ }^{6}$ Cape Heart Group, Hatter Institute for Cardiovascular Research in Africa, University of Cape Town, Cape Town, South Africa

Contributors AD and APK conceived and designed the protocol. AD, APK and KS performed the literature search, selection and quality assessment of the articles and extraction of the data. AD, APK, FT and KS interpreted the data. $A Q$ wrote the first draft of the manuscript. AD, APK, KS and FT contributed to the writing of the manuscript and agreed with manuscript results and conclusions. All authors read and approved the final manuscript.

Funding This research received no specific grant from any funding agency in the public, commercial or not-for-profit sectors.

Competing interests None.

Provenance and peer review Not commissioned; externally peer reviewed.

Data sharing statement No additional data are available.

Open Access This is an Open Access article distributed in accordance with the Creative Commons Attribution Non Commercial (CC BY-NC 3.0) license, which permits others to distribute, remix, adapt, build upon this work noncommercially, and license their derivative works on different terms, provided the original work is properly cited and the use is non-commercial. See: http:// creativecommons.org/licenses/by-nc/3.0/

\section{REFERENCES}

1. Fang JC, DeMarco T, Givertz MM, et al. World Health Organization Pulmonary Hypertension group 2: pulmonary hypertension due to left heart disease in the adult-a summary statement from the Pulmonary Hypertension Council of the International Society for Heart and Lung Transplantation. J Heart Lung Transplant 2012:31:913-33.

2. Simonneau G, Gatzoulis MA, Adatia I, et al. Updated clinical classification of pulmonary hypertension. J Am Coll Cardiol 2013;62 (25 Suppl):D34-41.

3. Guazzi M, Borlaug BA. Pulmonary hypertension due to left heart disease. Circulation 2012;126:975-90.
4. Haddad F, Kudelko K, Mercier O, et al. Pulmonary hypertension associated with left heart disease: characteristics, emerging concepts, and treatment strategies. Prog Cardiovasc Dis 2011:54:154-67.

5. Segers VF, Brutsaert DL, De Keulenaer GW. Pulmonary hypertension and right heart failure in heart failure with preserved left ventricular ejection fraction: pathophysiology and natural history. Curr Opin Cardiol 2012;27:273-80.

6. Vachiery JL, Adir Y, Barbera JA, et al. Pulmonary hypertension due to left heart diseases. J Am Coll Cardiol 2013;62(25 Suppl):D100-8.

7. Parker MW, Mittleman MA, Waksmonski CA, et al. Pulmonary hypertension and long-term mortality in aortic and mitral regurgitation. Am J Med 2010;123:1043-8.

8. Adhyapak SM. Effect of right ventricular function and pulmonary pressures on heart failure prognosis. Prev Cardiol 2010;13:72-7.

9. Merlos P, Nunez J, Sanchis J, et al. Echocardiographic estimation of pulmonary arterial systolic pressure in acute heart failure. Prognostic implications. Eur J Intern Med 2013;24:562-7.

10. Agarwal R, Shah SJ, Foreman AJ, et al. Risk assessment in pulmonary hypertension associated with heart failure and preserved ejection fraction. $J$ Heart Lung Transplant 2012;31:467-77.

11. Agarwal R. Prevalence, determinants and prognosis of pulmonary hypertension among hemodialysis patients. Nephrol Dial Transplant 2012;27:3908-14

12. Aronson D, Eitan A, Dragu R, et al. Relationship between reactive pulmonary hypertension and mortality in patients with acute decompensated heart failure. Circ Heart Fail 2011;4:644-50.

13. Bursi F, McNallan SM, Redfield MM, et al. Pulmonary pressures and death in heart failure: a community study. J Am Coll Cardiol 2012;59:222-31.

14. Strange G, Playford D, Stewart S, et al. Pulmonary hypertension: prevalence and mortality in the Armadale echocardiography cohort. Heart 2012;98:1805-11.

15. Mutlak D, Aronson D, Carasso S, et al. Frequency, determinants and outcome of pulmonary hypertension in patients with aortic valve stenosis. Am J Med Sci 2012;343:397-401.

16. Tatebe $\mathrm{S}$, Fukumoto $\mathrm{Y}$, Sugimura $\mathrm{K}$, et al. Clinical significance of reactive post-capillary pulmonary hypertension in patients with left heart disease. Circ J 2012;76:1235-44.

17. Stern J, Heist EK, Murray L, et al. Elevated estimated pulmonary artery systolic pressure is associated with an adverse clinical outcome in patients receiving cardiac resynchronization therapy. Pacing Clin Electrophysiol 2007;30:603-7.

18. Lee WT, Peacock AJ, Johnson MK. The role of per cent predicted 6-min walk distance in pulmonary arterial hypertension. Eur Respir J 2010;36:1294-301.

19. Moller JE, Hillis GS, Oh JK, et al. Prognostic importance of secondary pulmonary hypertension after acute myocardial infarction. Am J Cardiol 2005;96:199-203.

20. Cappola TP, Felker GM, Kao WH, et al. Pulmonary hypertension and risk of death in cardiomyopathy: patients with myocarditis are at higher risk. Circulation 2002;105:1663-8.

21. Szwejkowski BR, Elder DH, Shearer F, et al. Pulmonary hypertension predicts all-cause mortality in patients with heart failure: a retrospective cohort study. Eur J Heart Fail 2012;14:162-7.

22. Abramson SV, Burke JF, Kelly JJ Jr, et al. Pulmonary hypertension predicts mortality and morbidity in patients with dilated cardiomyopathy. Ann Intern Med 1992;116:888-95.

23. Kjaergaard J, Akkan D, Iversen KK, et al. Prognostic importance of pulmonary hypertension in patients with heart failure. $\mathrm{Am} J$ Cardiol 2007;99:1146-50.

24. Shalaby A, Voigt A, El-Saed A, et al. Usefulness of Pulmonary Artery Pressure by Echocardiography to Predict Outcome in Patients Receiving Cardiac Resynchronization Therapy Heart Failure. Am J Cardiol 2008;101:238-41.

25. Damy T, Goode KM, Kallvikbacka-Bennett A, et al. Determinants and prognostic value of pulmonary arterial pressure in patients with chronic heart failure. Eur Heart $J$ 2010;31:2280-90.

26. Ristow B, Ali S, Ren X, et al. Elevated pulmonary artery pressure by Doppler echocardiography predicts hospitalization for heart failure and mortality in ambulatory stable coronary artery disease: the Heart and Soul Study. J Am Coll Cardiol 2007;49:43-9.

27. Grigioni F, Potena L, Galie N, et al. Prognostic implications of serial assessments of pulmonary hypertension in severe chronic heart failure. J Heart Lung Transplant 2006;25:1241-6.

28. Levine TB, Levine AB, Goldberg D, et al. Impact of medical therapy on pulmonary hypertension in patients with congestive heart failure awaiting cardiac transplantation. Am J Cardiol 1996;78:440-3.

29. Lam CSP, Roger VL, Rodeheffer RJ, et al. Pulmonary hypertension in heart failure with preserved ejection fraction: a community-based study. J Am Coll Cardiol 2009;53:1119-26. 
30. Khush KK, Tasissa G, Butler J, et al. Effect of pulmonary hypertension on clinical outcomes in advanced heart failure: Analysis of the Evaluation Study of Congestive Heart Failure and Pulmonary Artery Catheterization Effectiveness (ESCAPE) database. Am Heart J 2009;157:1026-34.

31. Ghio S, Gavazzi A, Campana C, et al. Independent and additive prognostic value of right ventricular systolic function and pulmonary artery pressure in patients with chronic heart failure. J Am Coll Cardiol 2001;37:183-8.

32. Wang D, Han Y, Zang $\mathrm{H}$, et al. Prognostic effects of pulmonary hypertension in patients undergoing cardiac resynchronization therapy. J Thorac Dis 2010;2:71-5.

33. Ghio S, Temporelli PL, Klersy C, et al. Prognostic relevance of a non-invasive evaluation of right ventricular function and pulmonary artery pressure in patients with chronic heart failure. Eur $\mathrm{J}$ Heart Fail 2013;15:408-14.

34. Naidoo DP, Mitha AS, Vythilingum S, et al. Pulmonary hypertension in aortic regurgitation: early surgical outcome. Q J Med 1991;80:589-95.

35. Fawzy ME, Hassan W, Stefadouros M, et al. Prevalence and fate of severe pulmonary hypertension in 559 consecutive patients with severe rheumatic mitral stenosis undergoing mitral balloon valvotomy. J Heart Valve Dis 2004;13:942-7; discussion 47-8.

36. Roselli EE, Abdel Azim A, Houghtaling PL, et al. Pulmonary hypertension is associated with worse early and late outcomes after aortic valve replacement: implications for transcatheter aortic valve replacement. J Thorac Cardiovasc Surg 2012;144:1067-74 e2.

37. Melby SJ, Moon MR, Lindman BR, et al. Impact of pulmonary hypertension on outcomes after aortic valve replacement for aortic valve stenosis. J Thorac Cardiovasc Surg 2011;141:1424-30.

38. Le Tourneau T, Richardson M, Juthier F, et al. Echocardiography predictors and prognostic value of pulmonary artery systolic pressure in chronic organic mitral regurgitation. Heart 2010;96:1311-17.

39. Kainuma $\mathrm{S}$, Taniguchi $\mathrm{K}$, Toda $\mathrm{K}$, et al. Pulmonary hypertension predicts adverse cardiac events after restrictive mitral annuloplasty for severe functional mitral regurgitation. $J$ Thorac Cardiovasc Surg 2011;142:783-92.

40. Barbieri A, Bursi F, Grigioni F, et al. Prognostic and therapeutic implications of pulmonary hypertension complicating degenerative mitral regurgitation due to flail leaflet: a multicenter long-term international study. Eur Heart J 2011;32:751-9.

41. Manners JM, Monro JL, Ross JK. Pulmonary hypertension in mitral valve disease: 56 surgical patients reviewed. Thorax 1977;32:691-6

42. Malouf JF, Enriquez-Sarano M, Pellikka PA, et al. Severe pulmonary hypertension in patients with severe aortic valve stenosis: clinical profile and prognostic implications. J Am Coll Cardiol 2002;40:789-95.

43. Khandhar S, Varadarajan P, Turk R, et al. Survival benefit of aortic valve replacement in patients with severe aortic regurgitation and pulmonary hypertension. Ann Thorac Surg 2009;88:752-6.

44. Zuern CS, Eick C, Rizas K, et al. Prognostic value of mild-to-moderate pulmonary hypertension in patients with severe aortic valve stenosis undergoing aortic valve replacement. Clin Res Cardiol 2012;101:81-8.

45. Ben-Dor I, Goldstein SA, Pichard AD, et al. Clinical profile, prognostic implication, and response to treatment of pulmonary hypertension in patients with severe aortic stenosis. Am J Cardiol 2011;107:1046-51.

46. Yang C, Li D, Mennett R, et al. The impact of pulmonary hypertension on outcomes of patients with low left ventricular ejection fraction: a propensity analysis. J Heart Valve Dis 2012;21:767-73.

47. Nozohoor S, Hyllen S, Meurling C, et al. Prognostic value of pulmonary hypertension in patients undergoing surgery for degenerative mitral valve disease with leaflet prolapse. J Card Surg 2012;27:668-75.

48. Ward C, Hancock BW. Extreme pulmonary hypertension caused by mitral valve disease. Natural history and results of surgery. $\mathrm{Br}$ Heart J 1975;37:74-8.

49. Ghoreishi M, Evans CF, DeFilippi CR, et al. Pulmonary hypertension adversely affects short- and long-term survival after mitral valve operation for mitral regurgitation: implications for timing of surgery. $J$ Thorac Cardiovasc Surg 2011;142:1439-52.

50. Cam A, Goel SS, Agarwal S, et al. Prognostic implications of pulmonary hypertension in patients with severe aortic stenosis. $J$ Thorac Cardiovasc Surg 2011;142:800-8.

51. Pai RG, Varadarajan $\mathrm{P}$, Kapoor $\mathrm{N}$, et al. Aortic valve replacement improves survival in severe aortic stenosis associated with severe pulmonary hypertension. Ann Thorac Surg 2007;84:80-5.

52. Hayden JA, Cote P, Bombardier C. Evaluation of the quality of prognosis studies in systematic reviews. Ann Intern Med 2006;144:427-37.

53. de Jonge RC, van Furth $A M$, Wassenaar M, et al. Predicting sequelae and death after bacterial meningitis in childhood: a systematic review of prognostic studies. BMC Infect Dis 2010;10:232.

54. Vahanian A, Alfieri O, Andreotti F, et al. [Guidelines on the management of valvular heart disease (version 2012). The Joint Task Force on the Management of Valvular Heart Disease of the European Society of Cardiology (ESC) and the European Association for Cardio-Thoracic Surgery (EACTS)]. G Ital Cardiol (Rome) 2013;14:167-214.

55. Goldstone AB, Chikwe J, Pinney SP, et al. Incidence, epidemiology, and prognosis of residual pulmonary hypertension after mitral valve repair for degenerative mitral regurgitation. $\mathrm{Am} J \mathrm{Cardiol}$ 2011;107:755-60.

56. Damasceno A, Mayosi BM, Sani M, et al. The causes, treatment and outcome of acute heart failure in 1006 Africans from 9 countries. Arch Intern Med 2012;172:1386-94.

57. Janda S, Shahidi N, Gin K, et al. Diagnostic accuracy of echocardiography for pulmonary hypertension: a systematic review and meta-analysis. Heart 2011;97:612-22. 